ACCEPTED MAY $21^{S T} 2016$

\title{
FINITE ELEMENT SIMULATION OF NONLINEAR CONVECTIVE HEAT AND MASS TRANSFER IN A MICROPOLAR FLUID-FILLED ENCLOSURE WITH RAYLEIGH NUMBER EFFECTS
}

\author{
R. Bhargava ${ }^{1}$, S. Sharma ${ }^{1}$ P.Bhargava ${ }^{2}$, O. Anwar Bég ${ }^{3 *}$ and A. Kadir ${ }^{3}$ \\ ${ }^{1}$ Department of Mathematics, Indian Institute of Technology, Roorkee, India. \\ ${ }^{2}$ Fluid Mechanics, Department of Civil Engineering, Indian Institute of Technology, Roorkee, India. \\ ${ }^{3}$ Fluid Mechanics, Spray Research Group, School of Computing, Science and Engineering, G77, \\ Newton Building, University of Salford, Manchester, M54WT, UK.
}

\begin{abstract}
:
A mathematical model is presented to study the double-diffusive convective heat and mass transfer of a micropolar biofluid in a rectangular enclosure, as a model of transport phenomena in a bioreactor. The vertical walls of the enclosure are maintained at constant but different temperatures and concentrations. The conservation equations for linear momentum, angular momentum, energy and species concentration are formulated subject to appropriate boundary conditions and solved using both finite element and finite difference numerical techniques. Results are shown to be in excellent agreement between these methods. Several special cases of the flow regime are discussed. The distributions for streamline, isotemperature, isoconcentration and (isomicrorotation) are presented graphically for different Lewis number, buoyancy parameter, micropolar vortex viscosity parameter, gyration viscosity parameter, Rayleigh number, Prandtl number and micro-inertia parameter. Micropolar material parameters are shown to considerably influence the flow regime. The flow model has important applications in hybrid aerobic bioreactor systems exploiting rheological suspensions e.g. fermentation.
\end{abstract}

Key words: Double-diffusive heat and mass transfer; micropolar biofluid; diffusion; enclosure; Lewis number; vortex viscosity; iso-micro-rotation; Rayleigh number; numerical solutions; bioreactors.

*Corresponding author-Email: O.A.Beg@salford.ac.uk;gortoab@gmail.com

\section{INTRODUCTION}

Process engineering embraces many diverse technologies including materials manufacture, extrusion and increasingly synthesis of novel agents in bioreactors. Bioreactors are also an 
important device in medical biotechnology [1]. Working systems employ a variety of circulating biofluids which exhibit a diverse range of rheological and thermofluid characteristics. While mass transfer is an intrinsic component of bioreactor design [2], heat transfer is preferred in systems which are aerobic [3]. The fluid mechanics [4] of such systems involves double-diffusive natural convective heat and mass transfer in enclosures, a subject of interest in other disciplines including electronics, fuel cells, fire dynamics and solar cell technologies. Many Newtonian studies of enclosure (cavity) flows have been reported. A seminal study of double diffusive convection with combined driving forces was presented by Ostrach [5]. Makham and Rosenberger [6] studied the convective heat and mass transfer in crystal growth regimes in rectangular cavities. Bergman et al. [7] reported on the mixing hydrodynamics and growth processes in double-diffusive geophysical convection. Raganathan and Viskanta [8] studied analytically and computationally the binary free convection in a square cavity with combined thermal and species gradients. Nishimura et al. [9] also investigated the solidification of binary systems in double-diffusive heat and mass transfer flows. Bejan et al. [10] considered natural convection heat and mass transfer in a rectangular cavity subjected to various boundary conditions. Using both analytical and numerical techniques natural convection within a porous layer subjected to heat and mass fluxes in the horizontal direction was studied for a wide range of parameter values. A numerical study was later conducted by Lin [11] to analyze the transient natural convection heat and mass transfer in a square enclosure. Influences of the governing parameters on the unsteady variations of Nusselt and Sherwood numbers were examined and discussed in detail. Compared to such configurations, few investigations have considered the case where cross gradients of temperature and concentration are imposed. For this kind of boundary condition, numerical simulations of thermosolutal natural convection in a horizontal porous cavity were reported by Mohamad and Bennacer [12]. In their study, the porous matrix is heated and cooled along the vertical walls while concentration gradients are imposed vertically. They identified flow regimes for the cases of thermal and solutal dominating flows. Later they extended this study [13] to numerically model two- and three-dimensional thermosolutal convection in a horizontal enclosure filled with a saturated porous medium and subjected to cross gradients of temperature and concentration. Khanafer and Chamkha [14] used the ADI scheme to investigate the mixed convection flow in a Darcian lid-driven enclosure. Khanafer and Vafai [15] more recently studied the double-diffusive composite convection in a square enclosure containing a Darcy-Forcheimmer porous medium using finite volume and ADI methods.

All the above studies have been confined to Newtonian fluids. In numerous biomedical systems, working fluids (plasma, blood, anti-bacterial agents etc) exhibit non-Newtonian shear stress-strain 
characteristics and also complex micro-rheology. The Navier-Stokes viscous flow model is incapable of simulating such micro-structural characteristics, and biofluids therefore require a more sophisticated model for their accurate analysis. In this connection Eringen [16] in 1966 proposed the theory of micropolar fluids, in which the local effects arising from microstructure and intrinsic motions of the fluid elements were taken into account. While significant applications of this robust model have been reported in the context of biomechanics, chemical engineering heat transfer past bodies and channel flows, relatively few studies have emerged considering the convection of micropolar fluids in enclosures, including bioreactors. Several important investigations considering only convective heat transfer have been communicated. Jena and Bhattacharyya [17] provided one of the first analyses of micropolar thermal convection in a rectangular box heated from below. They used the Galerkin method and obtained critical Rayleigh numbers for various material parameters. Natural convection of micropolar fluids in a square cavity with differentially heated end walls was investigated later by Chen and Hsu [18] and Hsu and Chen [19]. In the former study they indicated the strong influence of micropolar parameter on the flow regime compared with a Newtonian fluid. In the latter they used a vorticity model formulation for two-dimensional convection of the form, $\Omega=-\nabla^{2} \Psi$ where $\Omega$ is the dimensionless vorticity and $\Psi$ is the dimensionless stream function. They used a cubic spline numerical scheme to show that the average Nusselt number is significantly lower for a micropolar fluid than for Newtonian fluid. While heat transfer rates were shown to be depressed by an increase in vortex viscosity parameter, heat flux infact was shown to be increased by micropolarity of the fluid. Several other studies of micropolar fluids in enclosed geometrical systems have also been reported. Chiu et al. [20] used an alternating direction implicit solver to examine the unsteady free convection of micropolar fluids in concentric spherical annuli.

These studies of micropolar convection in enclosed geometries were all restricted to only heat transfer. The coupled problem of mass transfer is fundamental to bioreactor systems. Important studies of relevance to this area are Srinivas and Murthy [21] who studied entropy generation in micropolar transport in porous media and Murthy et al. [22] who examined mciropolar hydrodynamics in stratified porous systems.

In the current study we consider double-diffusive heat and mass transfer of micropolar bio-suspension in an enclosure, as a two-dimensional simulation of transport in a bioreactor. The coupled differential conservation equations for linear momentum, microrotation (angular momentum), energy and concentration are solved numerically using both the finite element method and a finite difference method. Both methods have been applied extensively by the authors for over a decade in a wide variety of fluid dynamics problems. Contours are plotted for 
different values of buoyancy parameter, Lewis number, micropolar material parameters and also Rayleigh and Prandtl numbers, and the results are interpreted at length.

\section{MATHEMATICAL FORMULATION}

We study a two-dimensional enclosure (see figure 1) of height $H^{\prime}$ and width $L^{\prime}$. It is assumed that the third dimension of the cavity is large enough so that the transport processes can be assumed to be two-dimensional in nature. The vertical walls of the enclosure are subjected to temperature $T_{h}{ }^{\prime}$ and $T c^{\prime}$ at the vertical left and right walls, respectively while the adiabatic boundary conditions are applied at upper and horizontal walls. Heat conduction in the enclosure walls is neglected. The horizontal surfaces are subjected to a fixed concentration, viz a high concentration at the bottom $\left(S_{h}{ }^{\prime}\right)$ and low concentration $\left(S_{1}{ }^{\prime}\right)$ at the upper boundary, with adiabatic concentration at the left and right walls. It is assumed that the biofluid is an incompressible micropolar (rheological suspension) fluid and laminar flow is maintained. The constitutive equations for a micropolar fluid in vectorial form are, following Eringen [23]:

\section{Conservation of Mass}

$$
\frac{\partial \rho}{\partial t}+\nabla \cdot(\rho \mathbf{V})=0
$$

\section{Conservation of Translational Momentum}

$(\lambda+2 \mu+\chi) \nabla \nabla \bullet \boldsymbol{V}-(\mu+\chi) \nabla \times \nabla \times \boldsymbol{V}+\chi \nabla \times \boldsymbol{M}-\nabla \boldsymbol{P}+\rho f=\frac{\partial \mathbf{V}}{\partial t}-\boldsymbol{V} \times(\nabla \times \boldsymbol{V})+\frac{1}{2} \rho \nabla \mathbf{V}^{2}$

\section{Conservation of Angular Momentum (Micro-rotation)}

$$
\left(\alpha^{*}+\beta+\gamma\right) \nabla \nabla \bullet \mathbf{M}-\gamma \nabla \times \nabla \times \mathbf{M}+\chi \nabla \times \mathbf{V}-2 \chi \mathbf{M}+\rho \mathbf{l}=\rho j \dot{\mathbf{M}}
$$

where $\boldsymbol{P}$ is the hydrodynamic pressure and $\lambda, \mu, \chi, \alpha^{*}, \beta$ and $\gamma$ are viscosity coefficients of micropolar fluids, $\boldsymbol{V}=$ translational velocity vector, $\boldsymbol{M}$ is angular velocity (microrotation) vector and $\rho$ the mass density of micropolar fluid. In the micropolar model theory we are only concerned with two independent kinematical vector fields, namely the velocity vector field (familiar from Navier-Stokes theory) and the axial vector field which simulates the spin or the microrotations of 
the micropolar fluid particles, these being assumed non-deformable i.e. rigid. The thermophysical properties of the micropolar fluid are assumed to be constant, except density variation in the buoyancy terms, which is assumed to follow a linear variation with local temperature and concentration, in accordance with the Boussinesq approximation. This can be mathematically expressed as follows:

$$
\rho(T, S)=\rho_{0}{ }^{\prime}\left[1-\beta_{T}\left(T^{\prime}-T_{0}{ }^{\prime}\right)-\beta_{S}\left(S^{\prime}-S_{0}{ }^{\prime}\right)\right]
$$

where $S_{0}{ }^{\prime}$ and $T_{0}{ }^{\prime}$ are reference concentration and temperature at $x^{\prime}=0, y^{\prime}=0$. In (4) $\beta_{T}$ and $\beta_{S}$ designate the coefficients of thermal and species expansion, which are defined as follows:

$$
\begin{aligned}
& \beta_{T}=-\frac{1}{\rho_{o}}\left[\frac{\partial \rho}{\partial T}\right]_{P, C} \\
& \beta_{S}=-\frac{1}{\rho_{o}}\left[\frac{\partial \rho}{\partial C}\right]_{P, T}
\end{aligned}
$$

The conservation equations for micropolar double-diffusive convection in the enclosure can be shown to take the following form in an $\mathrm{x}^{\prime}, \mathrm{y}^{\prime}$ coordinate system, as illustrated in figure 1 , for vanishing pressure gradient (the flow is thermally- and solutally-driven, not pressuredriven):

$$
\begin{gathered}
\frac{\partial u^{\prime}}{\partial x^{\prime}}+\frac{\partial v^{\prime}}{\partial y^{\prime}}=0 \\
\frac{\partial v^{\prime}}{\partial x^{\prime}}-\frac{\partial u^{\prime}}{\partial y^{\prime}}=\frac{g K}{v}\left(\beta_{T} \frac{\partial T^{\prime}}{\partial x^{\prime}}+\beta_{s} \frac{\partial S^{\prime}}{\partial x^{\prime}}\right) \\
u^{\prime} \frac{\partial T^{\prime}}{\partial x^{\prime}}+v^{\prime} \frac{\partial T^{\prime}}{\partial y^{\prime}}=\alpha\left(\frac{\partial^{2} T^{\prime}}{\partial x^{\prime 2}}+\frac{\partial^{2} T^{\prime}}{\partial y^{\prime 2}}\right) \\
u^{\prime} \frac{\partial S^{\prime}}{\partial x^{\prime}}+v^{\prime} \frac{\partial S^{\prime}}{\partial y^{\prime}}=D\left(\frac{\partial^{2} S^{\prime}}{\partial x^{\prime 2}}+\frac{\partial^{2} S^{\prime}}{\partial y^{\prime 2}}\right) \\
u^{\prime} \frac{\partial G^{\prime}}{\partial x^{\prime}}+v^{\prime} \frac{\partial G^{\prime}}{\partial y^{\prime}}=\frac{K_{v}}{\rho j}\left(\frac{\partial^{2} \varphi^{\prime}}{\partial x^{\prime 2}}+\frac{\partial^{2} \varphi^{\prime}}{\partial y^{\prime 2}}-2 G^{\prime}\right)+\frac{\gamma}{\rho j}\left(\frac{\partial^{2} G^{\prime}}{\partial x^{\prime 2}}+\frac{\partial^{2} G^{\prime}}{\partial y^{\prime 2}}\right)
\end{gathered}
$$


where all parameters are defined in the nomenclature.

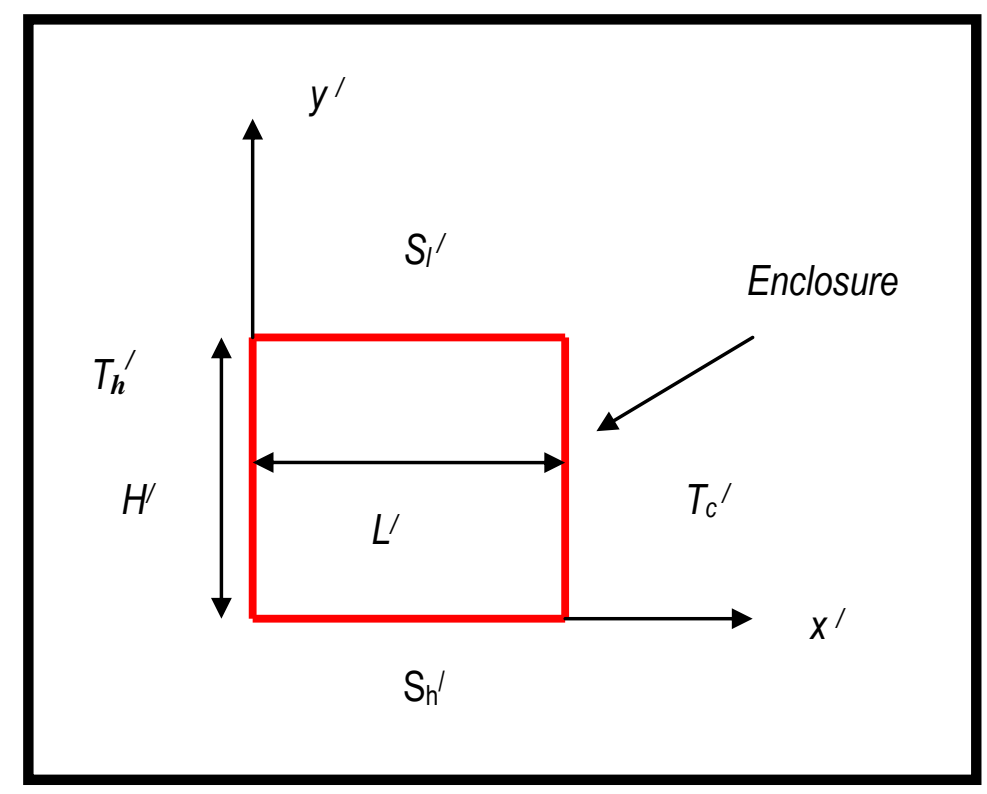

Figure 1: Physical model for micropolar double-diffusive convection in bioreactor enclosure

The equations are now transformed with the following non-dimensional variables, to facilitate a numerical solution, under appropriate boundary conditions:

$$
\begin{gathered}
(x, y)=\frac{\left(x^{\prime}, y^{\prime}\right)}{H^{\prime}}, \quad(u, v)=\frac{\left(u^{\prime}, v^{\prime}\right)}{\alpha / H^{\prime}}, \quad T=\frac{\left(T^{\prime}-T_{c}{ }^{\prime}\right)}{\Delta T^{\prime}}, \quad S=\frac{\left(S^{\prime}-S_{1}^{\prime}\right)}{\Delta S^{\prime}} \\
\varphi=\frac{\varphi^{\prime}}{\alpha}, \quad G=\frac{H^{\prime 2} G^{\prime}}{\alpha}
\end{gathered}
$$

The governing equations in non-dimensional form emerge as follows:

$$
\begin{aligned}
& u=-\frac{\partial \varphi}{\partial y}, \quad v=\frac{\partial \varphi}{\partial x} \\
& \nabla^{2} \varphi=R a\left(\frac{\partial T}{\partial x}+N \frac{\partial S}{\partial x}\right) \\
& \nabla^{2} T=\left(\frac{\partial(u T)}{\partial x}+\frac{\partial(v T)}{\partial y}\right)
\end{aligned}
$$




$$
\begin{gathered}
\nabla^{2} S=L e\left(\frac{\partial(u S)}{\partial x}+\frac{\partial(v S)}{\partial y}\right), \\
\frac{\partial(u G)}{\partial x}+\frac{\partial(v G)}{\partial y}=\operatorname{Pr} R B\left(\nabla^{2} \varphi-2 G\right)+\operatorname{Pr} \lambda \nabla^{2} G,
\end{gathered}
$$

where

$$
\begin{aligned}
& R a=\frac{g \beta_{T} K \Delta T^{\prime} H^{\prime}}{\alpha v}, \quad N=\frac{\beta_{S} \Delta S^{\prime}}{\beta_{T} \Delta T^{\prime}}, \quad L e=\frac{\alpha}{D}, \\
& \operatorname{Pr}=\frac{v}{\alpha}, \quad \lambda=\frac{\gamma}{j \mu}, B=\frac{H^{\prime 2}}{j}, R=\frac{K_{v}}{\mu}
\end{aligned}
$$

where $R a$ is the Rayleigh number, $N$ is the buoyancy ratio, $L e$ is the Lewis number, $P r$ is Prandtl number, $\lambda$ is a micropolar material parameter, $B$ is micro-inertia density parameter and $R$ is the micropolar vortex viscosity parameter. The boundary conditions for flow are the no-slip condition at the impermeable walls. Constant temperatures are imposed along the vertical walls with adiabatic conditions at the horizontal walls. Constant species concentration conditions are employed at the horizontal walls and zero mass fluxes at the vertical walls of the enclosure. Mathematically we can write the boundary conditions as follows:

$$
\begin{array}{lllll}
x=0, & \varphi=0, & T=0.5, & G=\frac{1 \partial v}{2 \partial x}, & \frac{\partial S}{\partial x}=0 ; \\
x=1, & \varphi=0, \quad T=-0.5, & G=\frac{1 \partial v}{2 \partial x} & \frac{\partial S}{\partial x}=0 ; \\
y=0, & \varphi=0, \quad \frac{\partial T}{\partial y}=0, & G=-\frac{1 \partial u}{2 \partial y} & S=0.5 ; \\
y=1, & \varphi=0, \quad \frac{\partial T}{\partial y}=0 ; & G=-\frac{1 \partial u}{2 \partial y} & S=-0.5 ;
\end{array}
$$

The average value of Nusselt and Sherwood numbers, which characterize the dimensionless heat transfer rate and mass transfer rate, respectively are evaluated at the horizontal and vertical walls, using the following expressions: 


$$
\begin{gathered}
N u=\int_{0}^{1}\left(\frac{\partial T}{\partial y}\right)_{y=1} d x \\
S h=\int_{0}^{1}\left(\frac{\partial S}{\partial x}\right)_{x=0} d y
\end{gathered}
$$

\section{NUMERICAL SOLUTIONS}

The set of equations (14) to (18) are highly nonlinear therefore this system of equations does not render analytical solutions. The finite element method has therefore been used to solve the model; additionally a finite difference method has been implemented to benchmark the finite element solutions. We shall discuss aspects of both numerical techniques now.

\subsection{Finite element method}

The finite element method is widely used for solving boundary value problems in fluid mechanics [24]. The technique has been employed by the authors in a number of areas of biofluid mechanics and bioheat and mass transfer, including pulsatile flows [25], nanofluids [26, 27] and biomagnetics [28]. The "infinite" flow domain is divided into smaller elements of finite dimensions termed 'finite elements'. The domain then constitutes an assemblage of these elements connected at a finite number of joints called 'nodes'. The concept of discretization as used in the finite difference method is then performed. Other features of the method include seeking continuous polynomial approximations of the solution over each element in term of nodal values, and assembly of element equations by imposing the inter-element continuity of the solution and balance of the inter-element forces. The major stages of the method are summarized below in flowchart 1 :

1. Division of the domain into linear elements, called the finite element mesh.

2. Generation of the element equations using variational formulations.

3. Assembly of the element equations as obtained in step (2).

4. Imposition of the boundary conditions to the equations obtained in step (3).

5. Solution of the assembled algebraic equations.

The assembled equations can be solved by any of the numerical technique viz. Gaussian elimination. The details of the steps given above can be found in [24].

\section{1-1 Variational Formulation: -}

The variational form associated with equations (14) to (18) over a typical square element is given by: 


$$
\begin{aligned}
& \int_{x_{e}}^{x_{e+1}} \int_{y_{e}}^{y_{e+1}} w_{1}\left(u+\frac{\partial \varphi}{\partial y}\right) d x d y=0 \\
& \int_{x_{e}}^{x_{e+1}} \int_{y_{e}}^{y_{e+1}} w_{2}\left(v-\frac{\partial \varphi}{\partial x}\right) d x d y=0 \\
& \int_{x_{e}}^{x_{e+1}} \int_{y_{e}}^{y_{e+1}} w_{3}\left(\nabla^{2} \varphi-R a\left(\frac{\partial T}{\partial x}+N \frac{\partial S}{\partial x}\right)\right) d x d y=0 \\
& \int_{x_{e}}^{x_{e+1}} \int_{y_{e}}^{y_{e+1}} w_{4}\left(\nabla^{2} T-\left(\frac{\partial(u T)}{\partial x}+\frac{\partial(v T)}{\partial y}\right)\right) d x d y=0 \\
& \int_{x_{e}}^{x_{e+1}} \int_{y_{e}}^{y_{e+1}} w_{5}\left(\nabla^{2} S-\left(\frac{\partial(u S)}{\partial x}+\frac{\partial(v S)}{\partial y}\right)\right) d x d y=0 \\
& \left.\int_{x_{e}}^{x_{e+1}} \int_{y_{e}}^{y_{e+1}} w_{6}\left(\frac{\partial(u G)}{\partial x}+\frac{\partial(v G)}{\partial y}-\operatorname{Pr} R B\left(\nabla^{2} \varphi-2 G\right)-\operatorname{Pr} \lambda \nabla^{2} G\right)\right) d x d y=0
\end{aligned}
$$

where $w_{1}, w_{2}, w_{3}, w_{4}, w_{5}$ and $w_{6}$ are arbitrary test function and may be viewed as the variation in $u, v, \varphi, T, S$ and $G$ respectively. All functions satisfy the homogeneous boundary conditions, as per theoretical requirements.

\section{1-2 Finite Element Formulation: -}

The flow domain defined as: $0 \leq x \leq 1$, and $0 \leq y \leq 1$ is discretized into square elements of same size. The finite element model is now obtained from the equations (23-28), by substituting finite element approximations of the form.

$$
\begin{array}{ll}
u=\sum_{j=1}^{4} u_{j} \psi_{j}, & v=\sum_{j=1}^{4} v_{j} \psi_{j}, \\
\varphi & =\sum_{j=1}^{4} \phi_{j} \psi_{j}, \quad T=\sum_{j=1}^{4} T_{j} \psi_{j}, \\
S & =\sum_{j=1}^{4} S_{j} \psi_{j}, \quad G=\sum_{j=1}^{4} G_{j} \psi_{j}
\end{array}
$$


with $w_{i}=\psi_{j}(i=1,2,3,4,5,6, j=1,2,3,4)$ and where $\psi_{1}, \psi_{2}, \psi_{3}$ and $\psi_{4}$ are the linear interpolation functions for a rectangular element $\Omega^{e}$ and these are defined as:

$$
\begin{aligned}
& \psi_{1}=\frac{\left(x_{e+1}-x\right)}{\left(x_{e+1}-x_{e}\right)} \frac{\left(y_{e+1}-y\right)}{\left(y_{e+1}-y_{e}\right)}, \\
& \psi_{2}=\frac{\left(x-x_{e}\right)}{\left(x_{e+1}-x_{e}\right)} \frac{\left(y_{e+1}-y\right)}{\left(y_{e+1}-y_{e}\right)}, \\
& \psi_{3}=\frac{\left(x-x_{e}\right)}{\left(x_{e+1}-x_{e}\right)} \frac{\left(y-y_{e}\right)}{\left(y_{e+1}-y_{e}\right)}, \\
& \psi_{4}=\frac{\left(x_{e+1}-x\right)}{\left(x_{e+1}-x_{e}\right)} \frac{\left(y-y_{e}\right)}{\left(y_{e+1}-y_{e}\right)}
\end{aligned}
$$

These equations are then written in matrix-vector form as:

$$
\left[\begin{array}{llll}
{\left[K^{11}\right]} & \left.\left[K^{12}\right]\left[K^{13}\right]\left[K^{14}\right]\left[K^{15}\right]\left[K^{16}\right]\right] \\
{\left[K^{21}\right]} & {\left[K^{22}\right]\left[K^{23}\right]\left[K^{24}\right]\left[K^{25}\right]\left[K^{26}\right]} \\
{\left[K^{31}\right]} & {\left[K^{32}\right]\left[K^{33}\right]\left[K^{34}\right]\left[K^{35}\right]\left[K^{36}\right]} \\
{\left[K^{41}\right]} & {\left[K^{42}\right]\left[K^{43}\right]\left[K^{44}\right]\left[K^{45}\right]\left[K^{46}\right]} \\
{\left[K^{51}\right]} & {\left[K^{52}\right]\left[K^{53}\right]\left[K^{54}\right]\left[K^{55}\right]\left[K^{56}\right]} \\
{\left[K^{61}\right]} & {\left[K^{62}\right]\left[K^{63}\right]\left[K^{64}\right]\left[K^{65}\right]\left[K^{66}\right]}
\end{array}\right]\left[\begin{array}{l}
\{\varphi\} \\
\{T\} \\
\{S\} \\
\{G\}
\end{array}\right]=\left[\begin{array}{l}
\left\{b^{1}\right\} \\
\left\{b^{2}\right\} \\
\left\{b^{3}\right\} \\
\left\{b^{4}\right\} \\
\left\{b^{5}\right\} \\
\left\{b^{6}\right\}
\end{array}\right\}
$$

where $\left[K^{m n}\right]$ and $\left[b^{m}\right] \quad(m, n=1,2,3,4,5,6)$ are defined as follows:

$$
\begin{aligned}
& K_{i j}^{11}=\int_{\Omega^{e}} \psi_{i} \psi_{j} d x d y, \quad K_{i j}^{13}=\int_{\Omega^{e}} \psi_{i} \frac{\partial \psi_{j}}{d y} d x d y, \\
& K_{i j}^{12}=K_{i j}^{14}=K_{i j}^{15}=0 \\
& K_{i j}^{22}=\int_{\Omega^{e}} \psi_{i} \psi_{j} d x d y, K_{i j}^{23}=-\int_{\Omega^{e}} \psi_{i} \frac{\partial \psi_{j}}{d y} d x d y, \\
& K_{i j}^{21}=K_{i j}^{24}=K_{i j}^{25}=0
\end{aligned}
$$




$$
\begin{aligned}
& K_{i j}^{33}=\int_{\Omega^{e}}\left(\frac{\partial \psi_{i}}{d x} \frac{\partial \psi_{j}}{d x}+\frac{\partial \psi_{i}}{d y} \frac{\partial \psi_{j}}{d y}\right) d x d y, \\
& K_{i j}^{34}=-R a \int_{\Omega^{e}} \psi_{i} \frac{\partial \psi_{j}}{d x} d x d y, K_{i j}^{31}=K_{i j}^{32}=0 \\
& K_{i j}^{34}=-R a N \int_{\Omega^{e}} \psi_{i} \frac{\partial \psi_{j}}{d x} d x d y
\end{aligned}
$$

$$
\begin{gathered}
\left.K_{i j}^{44}=-\int_{\Omega^{e}-\int_{\Omega^{e}}\left(\frac{\partial \psi_{i}}{d x} \frac{\partial \psi_{j}}{d x}+\frac{\partial \psi_{i}}{d y} \frac{\partial \psi_{j}}{d y}\right) d x d y} \frac{\partial \psi_{j}}{d x}+\bar{v} \psi_{i} \frac{\partial \psi_{j}}{d y}\right) d x d y \\
K_{i j}^{41}=K_{i j}^{42}=K_{i j}^{43}=K_{i j}^{45}=0
\end{gathered}
$$

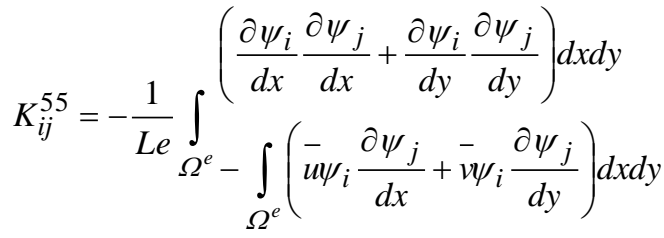

$$
\begin{aligned}
& K_{i j}^{61}=\operatorname{Pr} R B \int_{\Omega^{e}}\left(\frac{\partial \psi_{i}}{d x} \frac{\partial \psi_{j}}{d x}+\frac{\partial \psi_{i}}{d y} \frac{\partial \psi_{j}}{d y}\right) d x d y \\
& K_{i j}^{66}=\operatorname{Pr} \int_{\Omega^{e}}+\int_{\Omega^{e}}\left(\frac{\partial \psi_{i}}{d x} \frac{\partial \psi_{j}}{d x}+\frac{\partial \psi_{i}}{d y} \frac{\partial \psi_{j}}{d y}\right) d x d y \\
& +\operatorname{Pr} R B \int_{\Omega^{e}}\left(\psi_{i} \psi_{j}\right) d x d y \\
& \left.K_{i j}^{62}=K_{i j}^{63}=K_{i j}^{64} \frac{\partial \psi_{j}}{d y}\right) d x d y \\
& b_{i}^{1}=0, \quad K_{i j}^{6}=0, \\
& b_{i}^{3}=0, \quad b_{i}^{4}=0, \\
& b_{i}^{5}=0, b_{i}^{6}=-0
\end{aligned}
$$


and $\quad \bar{u}=\sum_{i=1}^{4} \psi_{i} u_{i}, \quad \bar{v}=\sum_{i=1}^{4} \psi_{i} v_{i}$. The whole domain is divided into 100 square elements of length 0.1. Each element is four-noded therefore whole domain contains 121 nodes. At each node 6 functions are to be evaluated; hence after assembly of the element equations we obtain a system of 726 equations which is non-linear therefore an iterative scheme is used for solving it. The system is linearized by incorporating the functions $\bar{u}$ and $\bar{v}$, which are assumed to be known. The system of linear equations has been solved using Gaussian elimination and an accuracy of 0.0005 has been maintained in the computations. It has been observed that in the same domain the accuracy is not affected even if the number of elements are increased, by decreasing the size of the elements. Further mesh refinement serves only to escalate compilation times.

\subsection{Finite difference method}

For the purposes of comparison, the same system of equations (14)-(18), subject to boundary conditions (20) is solved numerically using the finite difference method. This method is used for solving the ordinary as well as partial differential equations governing boundary value problem as well as initial value problem. By using the central difference approximations [29], the set of differential equations (14)-(18), can be discretized to give:

$$
\begin{gathered}
u(i, j)=-\left(\frac{\varphi(i, j+1)-\varphi(i, j-1)}{2 k e}\right) \quad, \quad v(i, j)=\left(\frac{\varphi(i+1, j)-\varphi(i-1, j)}{2 h e}\right) \\
\left(\frac{\varphi(i+1, j)-2 \varphi(i, j)+\varphi(i-1, j)}{h e^{2}}\right)+\left(\frac{\varphi(i, j+1)-2 \varphi(i, j)+\varphi(i, j-1)}{k e^{2}}\right) \\
=R a\left(\left(\frac{T(i+1, j)-T(i-1, j)}{2 h e}\right)+N\left(\frac{S(i+1, j)-S(i-1, j)}{2 h e}\right)\right)
\end{gathered}
$$

$$
\begin{gathered}
\left(\frac{T(i+1, j)-2 T(i, j)+T(i-1, j)}{h e^{2}}\right)+\left(\frac{T(i, j+1)-2 T(i, j)+T(i, j-1)}{k e^{2}}\right) \\
=u(i, j)\left(\frac{T(i+1, j)-T(i-1, j)}{2 h e}\right)+v(i, j)\left(\frac{T(i, j+1)-T(i, j-1)}{2 k e}\right) \\
\left(\frac{S(i+1, j)-2 S(i, j)+S(i-1, j)}{h e^{2}}\right)+\left(\frac{S(i, j+1)-2 S(i, j)+S(i, j-1)}{k e^{2}}\right) \\
=\operatorname{Le}\left(u(i, j)\left(\frac{S(i+1, j)-S(i-1, j)}{2 h e}\right)+v(i, j)\left(\frac{S(i, j+1)-S(i, j-1)}{2 k e}\right)\right)
\end{gathered}
$$




$$
\begin{aligned}
& \left(u(i, j)\left(\frac{G(i+1, j)-G(i-1, j)}{2 h e}\right)\right)+\left(v(i, j)\left(\frac{G(i, j+1)-G(i, j-1)}{2 k e}\right)\right) \\
& =\operatorname{Pr} R B\left(\left(\left(\frac{\varphi(i+1, j)-2 \varphi(i, j)+\varphi(i-1, j)}{h e^{2}}\right)+\left(\frac{\varphi(i, j+1)-2 \varphi(i, j)+\varphi(i, j-1)}{k e^{2}}\right)\right)-2 G(i, j)\right) \\
& \quad+\operatorname{Pr} \lambda\left(\left(\frac{G(i+1, j)-2 G(i, j)+G(i-1, j)}{h e^{2}}\right)+\left(\frac{G(i, j+1)-2 G(i, j)+G(i, j-1)}{k e^{2}}\right)\right)
\end{aligned}
$$

where he and ke denote the $\mathrm{x}$ and $\mathrm{y}$ direction step-lengths. Since the above equations are nonlinear and coupled hence they cannot be solved exactly. Therefore an iterative scheme is required to be used. Writing down the equations in the form:

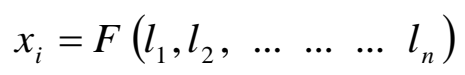

where each $l_{i}$ is the function of the variable $u_{i}, v_{i}, \varphi_{i}, T_{i}, S_{i}$ and $x_{i}$ is any of the variable $u_{i}, v_{i}, \varphi_{i}, T_{i}, S_{i}$. Now starting with initial guess values, new iterate values are obtained. This

process continues until the absolute error $\left|x_{i}-x_{i-1}\right|$ is less than the accuracy required. The condition of convergence of the scheme has been already checked before implementing the iterative scheme. Further excellent details on modern applications of the finite difference method are available in Sohail et al. [30] and Sohail et al. [31] where adapting of algorithms to capillary gravity wave and magnetic nanoscale flow problems have been considered. Therefore while the finite difference approach is classical, it is still very versatile and has significant abilities for solving nonlinear boundary value problems in transport phenomena.

\section{RESULTS AND DISCUSSION}

The controlling parameters which dictate the flow regime for double diffusive natural convection in an enclosure are the aspect ratio $A$, and the dimensionless numbers $\operatorname{Ra}, \operatorname{Pr}, N$, $L e, \lambda, \mathrm{B}$ and $\mathrm{R}$. We shall discuss the influence of each of these parameters in detail. Prandtl number $\operatorname{Pr}$ is fixed at 7.0 and Lewis number, Le at 10 corresponding to a model of salt diffusion in water, representative of brine-based bioreactors [3] and for an aspect ratio of 1.0 i.e. a square enclosure, unless otherwise stated. ( $\mathrm{Le}=1$ in oxygen).

Stable solutal stratified fluid resists flow evolution. On the other hand applying horizontal temperature gradient across the cavity initiates flow, even for a very small temperature gradient. Hence there is a competition between thermal and solutal buoyancy forces. As $N$ approaches zero, the thermal buoyancy drives the flow and formation of a longitudinal recirculated flow is 
expected for moderately high $R a$ parameter. As $N$ becomes much larger than unity, the flow is suppressed in the enclosure and diffusion dominates the heat and mass transfer. For $N$ of order unity, the effect of thermal and solutal buoyancy forces becomes of the same order and magnitude.

Figures 1-9 illustrate the influence of various parameters on the regime. Figures 1 to 4 demonstrate typical features of aiding double diffusive flow for buoyancy ratio $N$. Figures $1 \mathrm{a}$, $1 \mathrm{~b}, 1 \mathrm{c}$ and $1 \mathrm{~d}$ show the representative sequence of streamline isotherm pattern, iso-concentration and iso-microrotation pattern in a square domain for $N=0$ i.e. where buoyancy ratio is zero. Setting $N=0$ effectively de-couples the vorticity equation (15) from the species conservation equation (17).

\begin{tabular}{|c|c|c|c|c|}
\hline \multicolumn{5}{|c|}{$R a=100, \lambda=.50, \operatorname{Pr}=1.0, R=1 . B=.5, L e=.1$} \\
\hline$N$ & $-T^{\prime}(0)$ FEM & $-T^{\prime}(0) \mathrm{FDM}$ & $-S^{\prime}(0)(\mathrm{FEM})$ & $-S^{\prime}(0)(\mathrm{FDM})$ \\
\hline-0.50 & 1.0917 & 1.0923 & .595659 & .59247 \\
\hline 0.0 & 1.19425 & 1.19342 & .54887 & .54863 \\
\hline 0.50 & 1.32167 & 1.32540 & .50195 & .50174 \\
\hline 1.0 & 1.46554 & 1.46638 & .464043 & .46684 \\
\hline 5.0 & 2.02833 & 2.02549 & .664634 & .66045 \\
\hline 10.0 & 2.33136 & 2.33074 & 1.02901 & 1.02468 \\
\hline \multicolumn{5}{|c|}{$R a=100, \lambda=.50, \operatorname{Pr}=1.0, R=1 . B=.5, N=.1$} \\
\hline Le & $-T^{\prime}(0)(\mathrm{FEM})$ & $-T^{\prime}(0) \mathrm{FDM}$ & $-S^{\prime}(0)(\mathrm{FEM})$ & $-S^{\prime}(0)(\mathrm{FDM})$ \\
\hline .001 & 1.19437 & 1.19426 & .99364 & .99553 \\
\hline .1 & 1.20667 & 1.2058 & .936199 & .936886 \\
\hline 1.0 & 1.32167 & 1.32085 & .50195 & .501228 \\
\hline 5.0 & 1.29295 & 1.29478 & .941962 & .941324 \\
\hline 10.0 & 1.23751 & 1.23687 & 1.18054 & 1.18165 \\
\hline 20.0 & 1.19164 & 1.19320 & 1.192009 & 1.193117 \\
\hline
\end{tabular}

Table 1. Table for the rate of heat transfer $\left\{-T^{\prime}(0)\right\}$ and rate of mass transfer $\left\{-S^{\prime}(0)\right\}$ with different value of buoyancy parameter $N$ and Lewis number $L e$. 


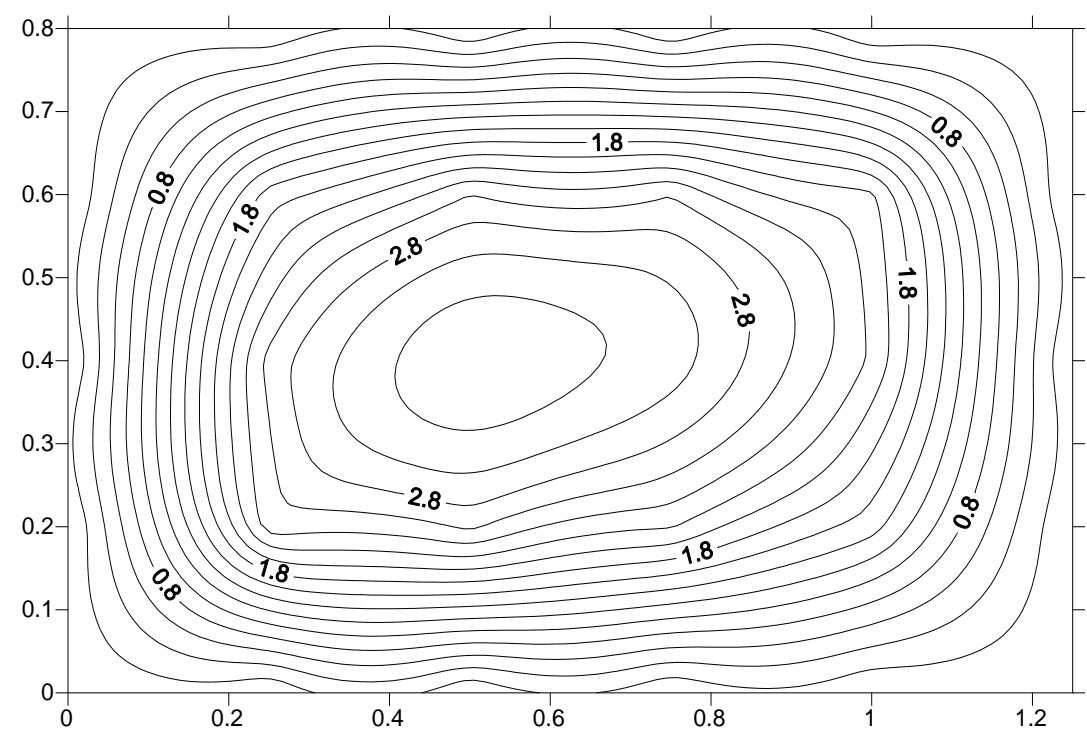

Figure 1a: Stream line profiles in the enclosure for $N=0$

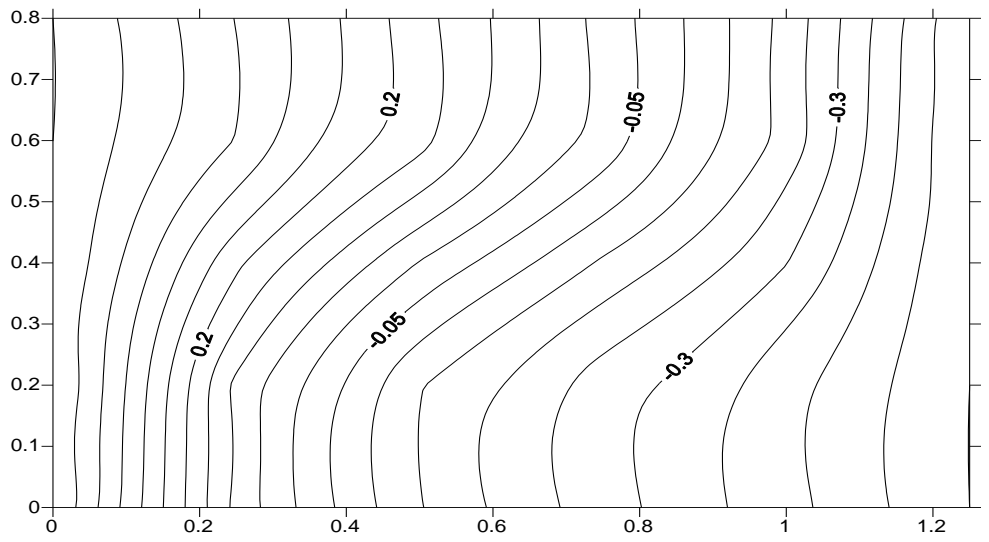

Fig.1.b : Iso-temperature profiles in enclosure for $N=0$

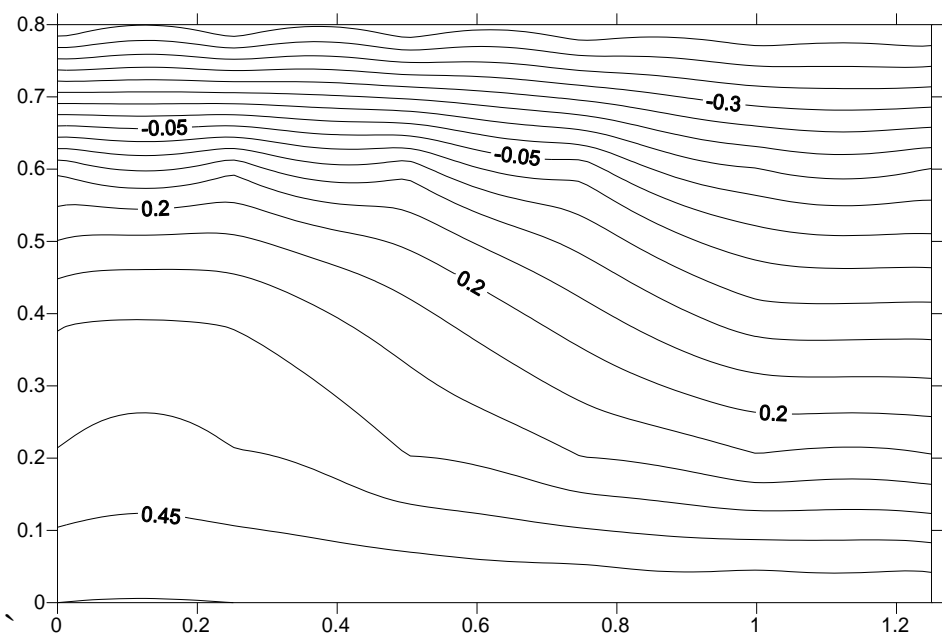

Fig 1c: Iso-concentration profiles for $N=0$ 


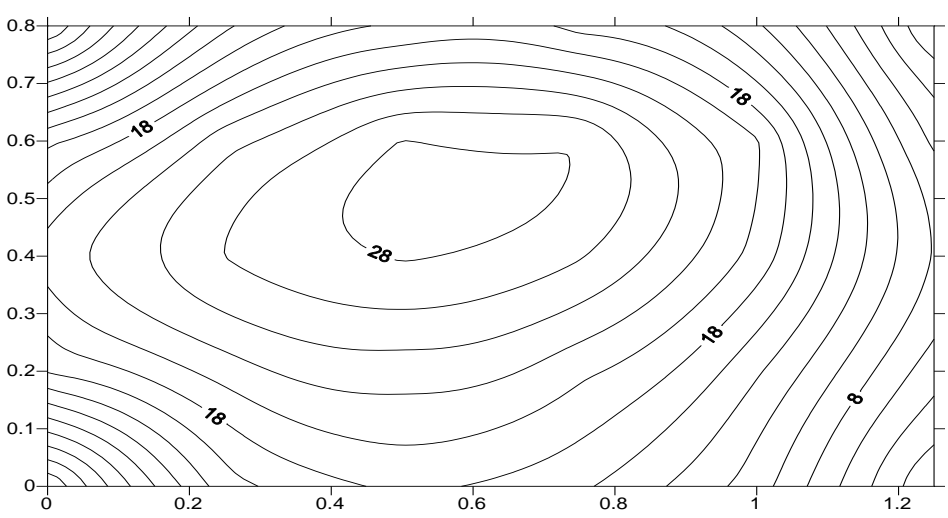

Fig.1.d : Iso-micro-rotation profiles for $N=0$

Figures 1a to 1d: $R a=100, L e=1, B=1 ., \lambda=1 ., R=1 ., N=0$

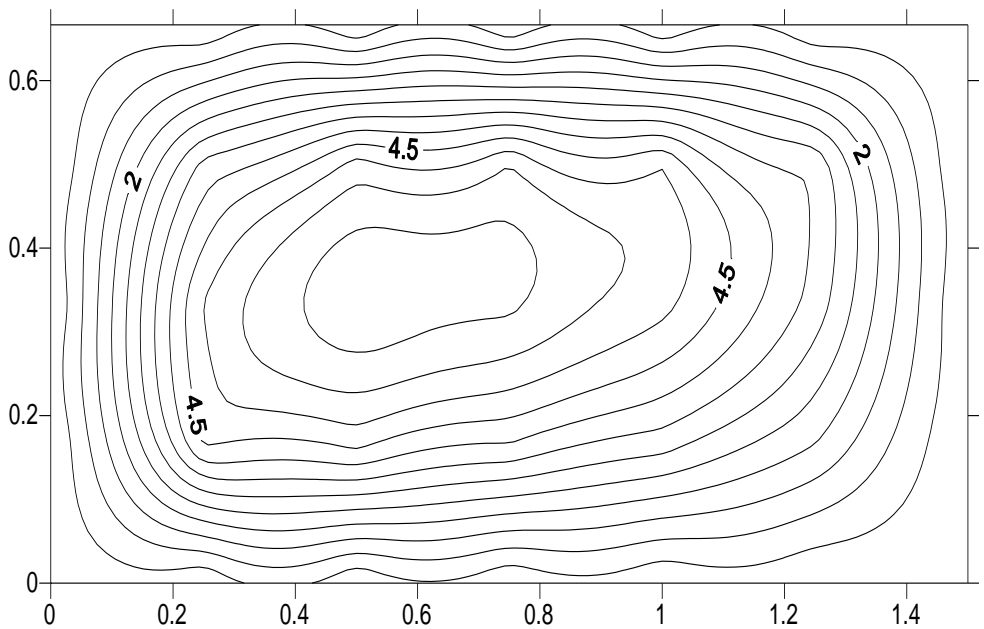

Figure 2a: Influence of $N$ on the streamline distribution

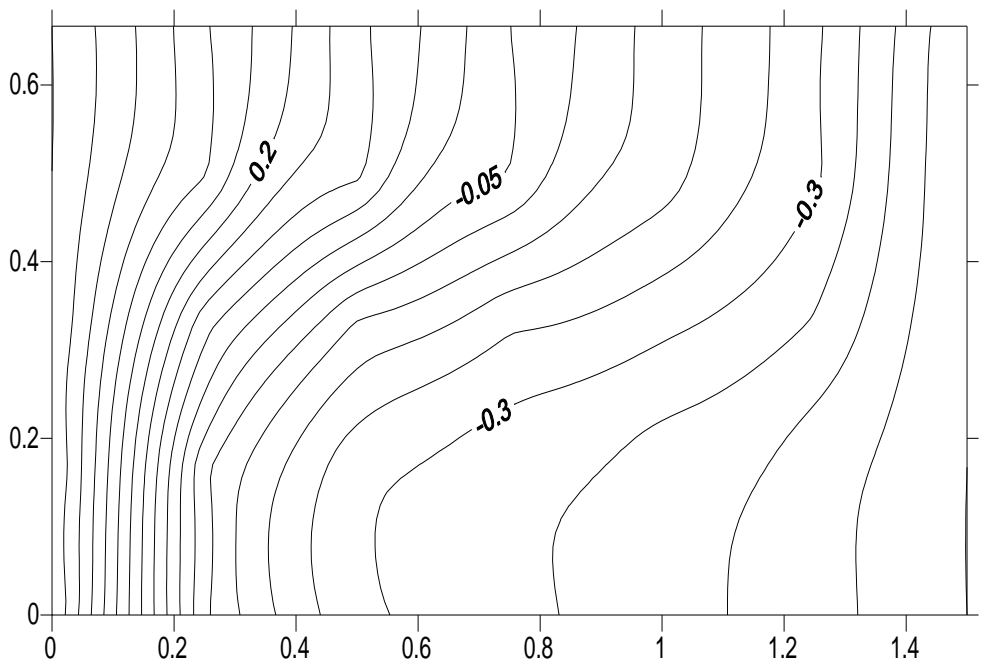

Figure 2b: Influence of $N$ on the iso-temperature distribution 


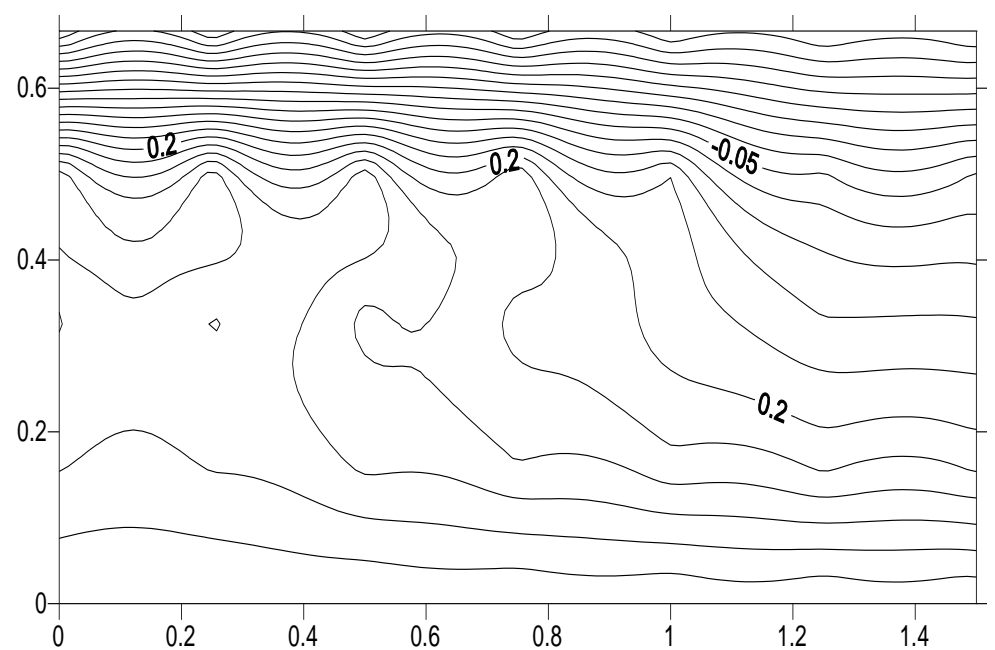

Figure 2c: Influence of $N$ on the iso-concentration distribution

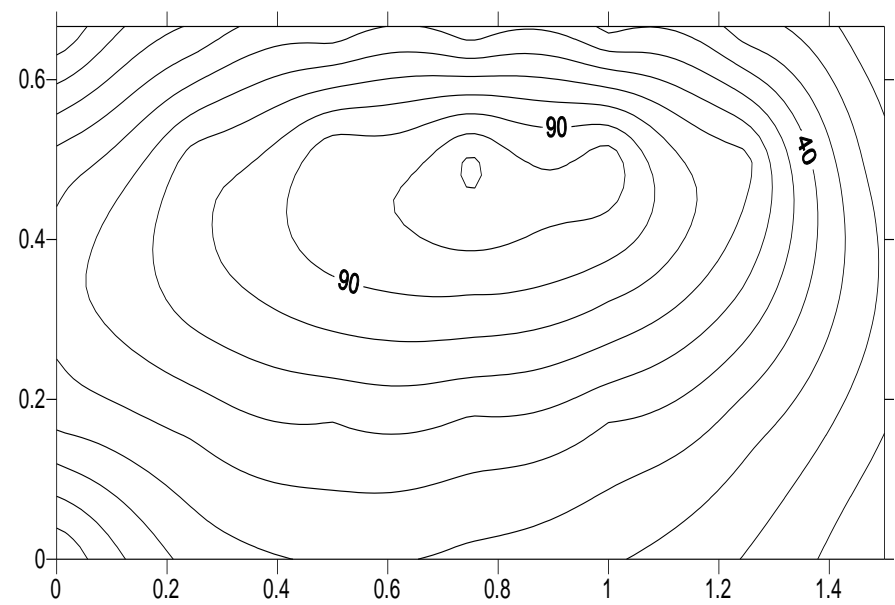

Fig.2.d Influence of $N$ on the iso-micro-rotation distribution

Figures 2 a to $2 d: \quad R a=100, L e=1, B=1 ., \lambda=1$., $R=1 ., N=1$ 


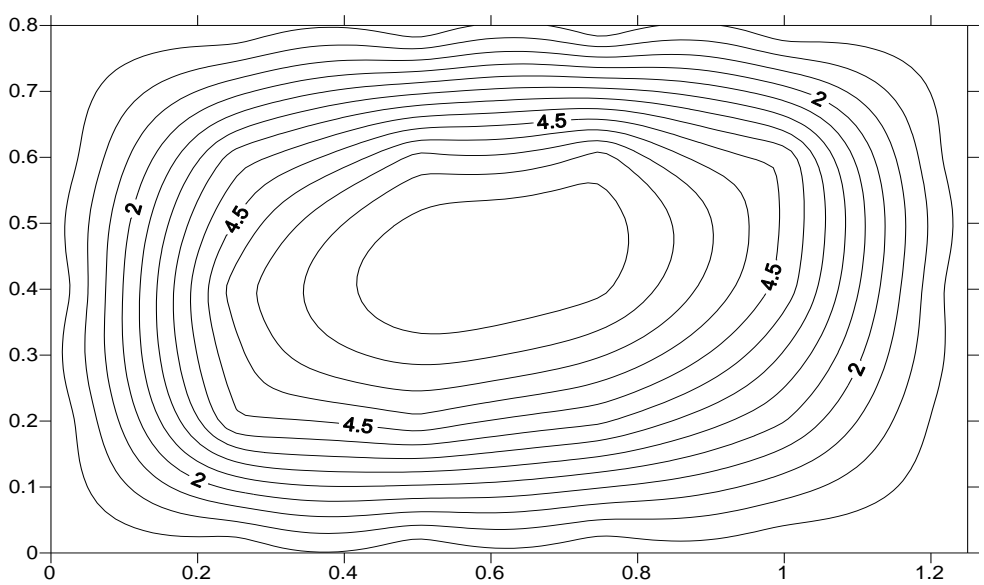

Figure 3a: Influence of larger $N$ on the streamline distribution

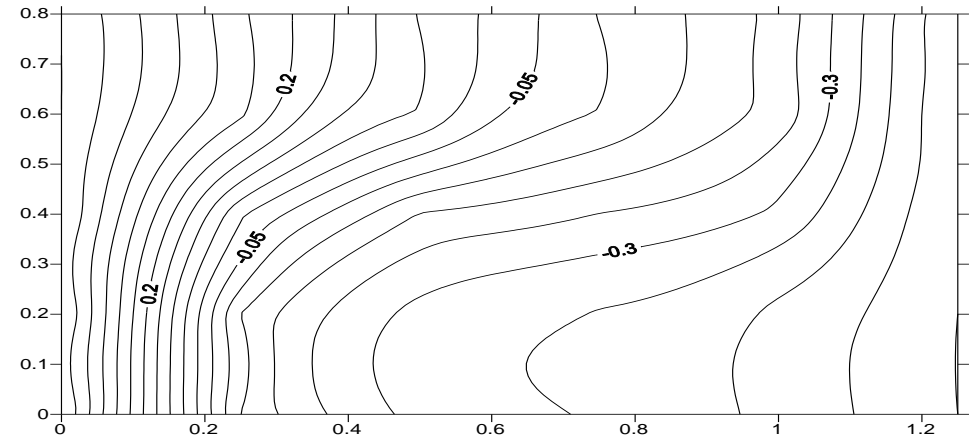

Figure 3.b : Influence of larger $\mathbf{N}$ on the iso-temperature distribution

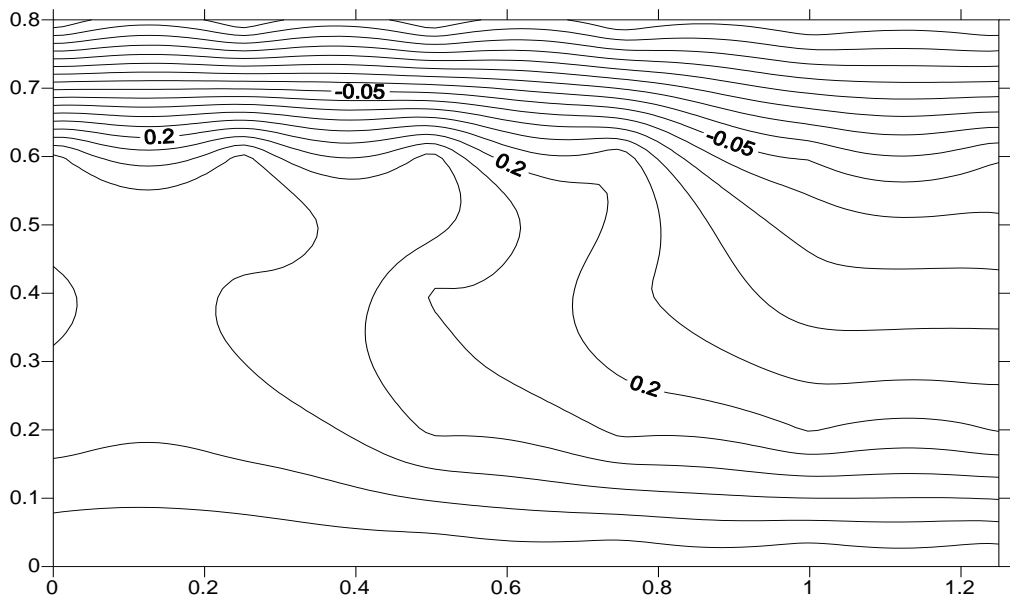

Figure 3c: Influence of larger $N$ on the iso-concentration distribution 


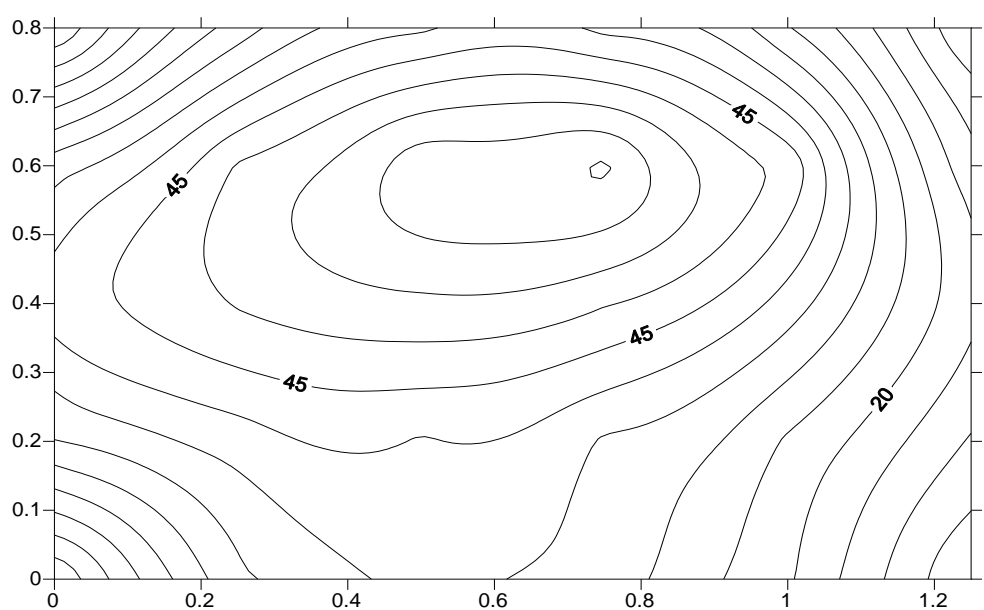

Figure 3d: Influence of larger $N$ on the iso-micro-rotation distribution

Figures $3 a$ to $3 d: R a=100, L e=1, B=1 ., \lambda=1 ., R=1 ., N=4$

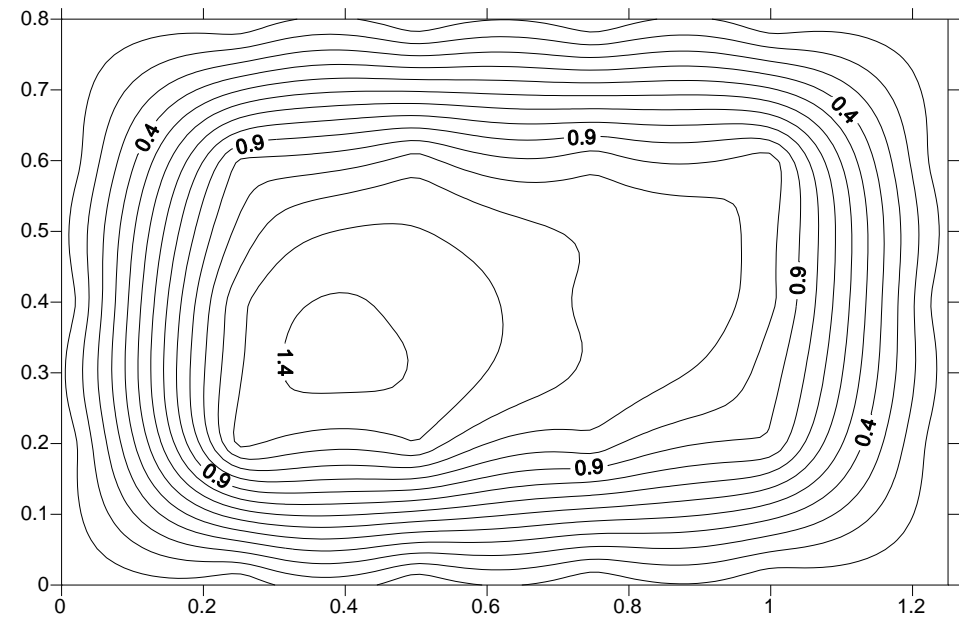

Figure 4a: Influence of negative $N$ on streamline distribution

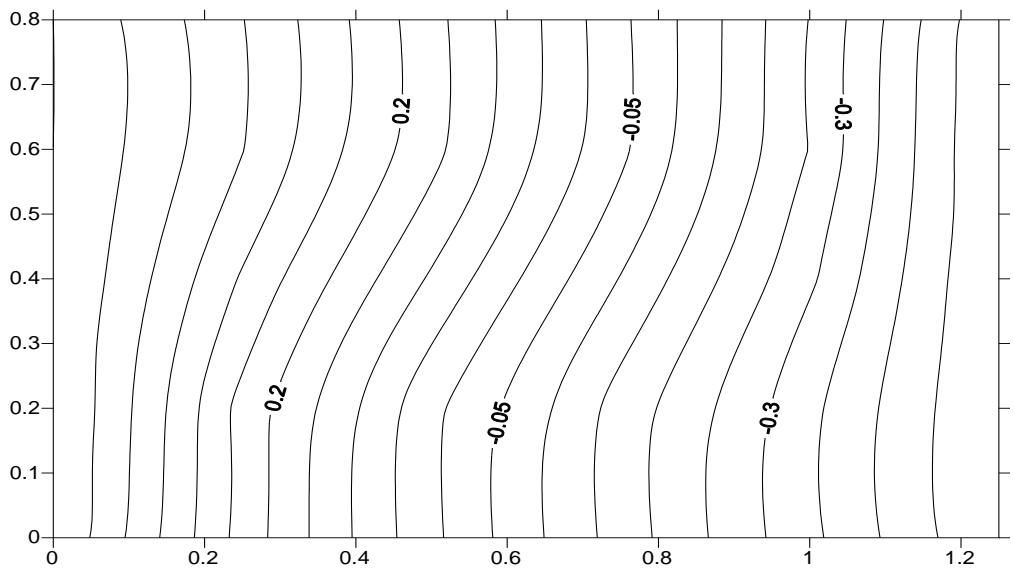

Figure 4b: Negative $N$ effects on iso-temperature distribution 


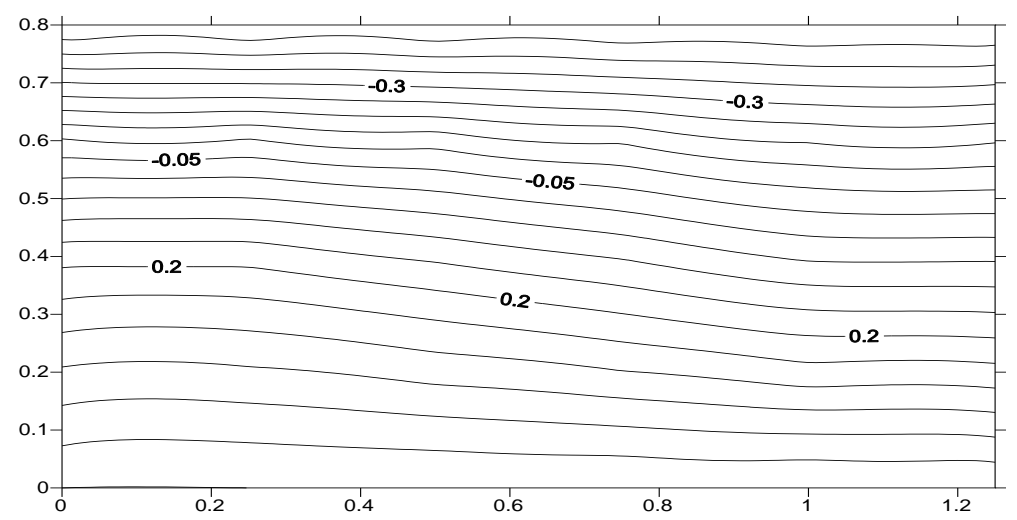

Figure 4c: Negative $N$ effects on iso-concentration distribution

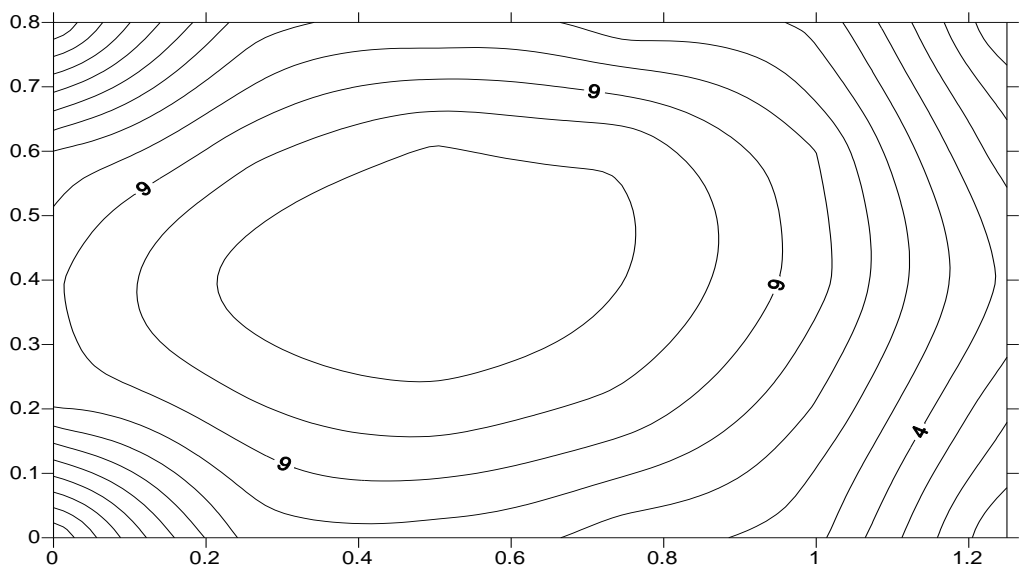

Figure 4d: Negative $N$ effects on iso-micro-rotation distribution

Figures 4a to d: $R a=100, L e=1, B=1 ., \lambda=1 ., R=1 ., N=-4$ 


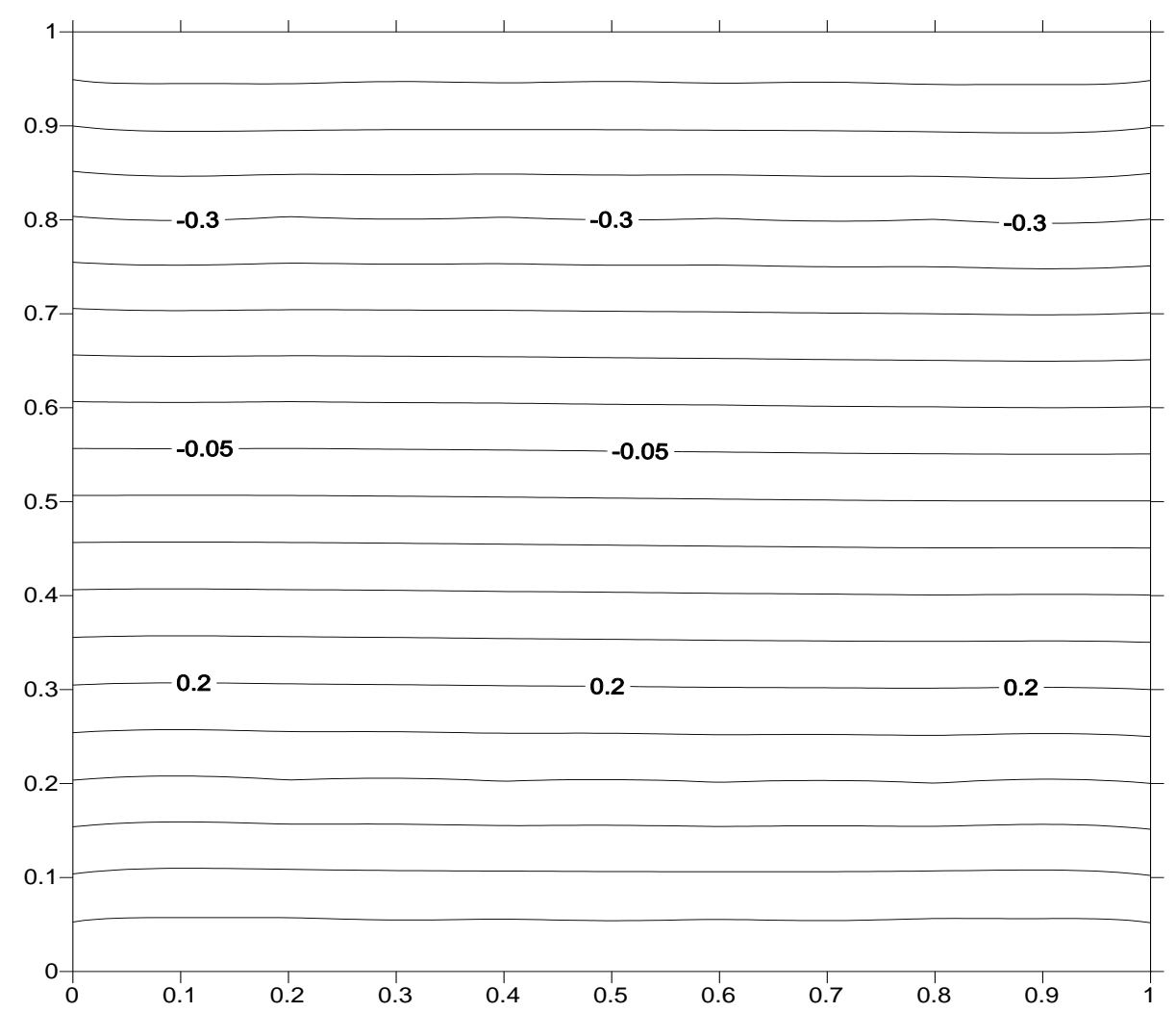

Figure 5a: Iso-concentration profile for Le $=0.01$

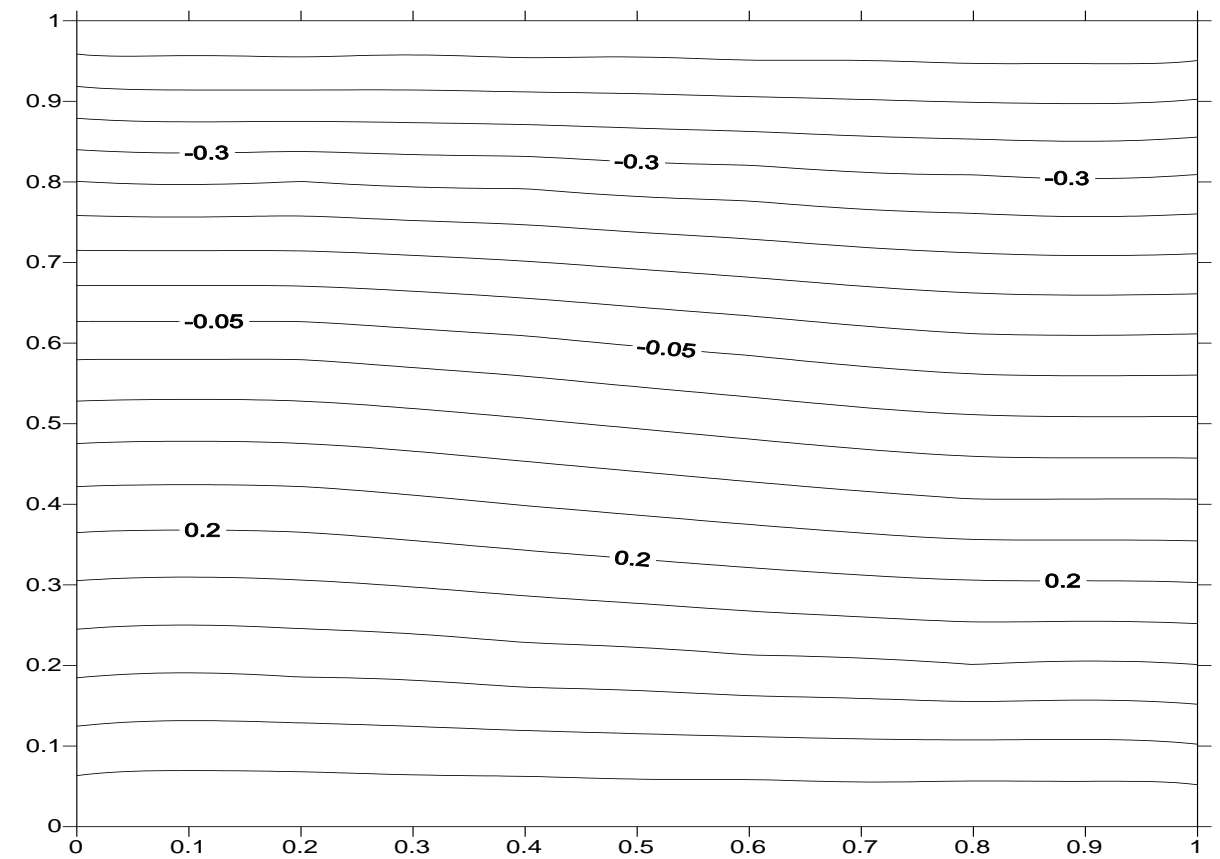

Figure 5 b: Iso-concentration profile for $L e=0.1$ 


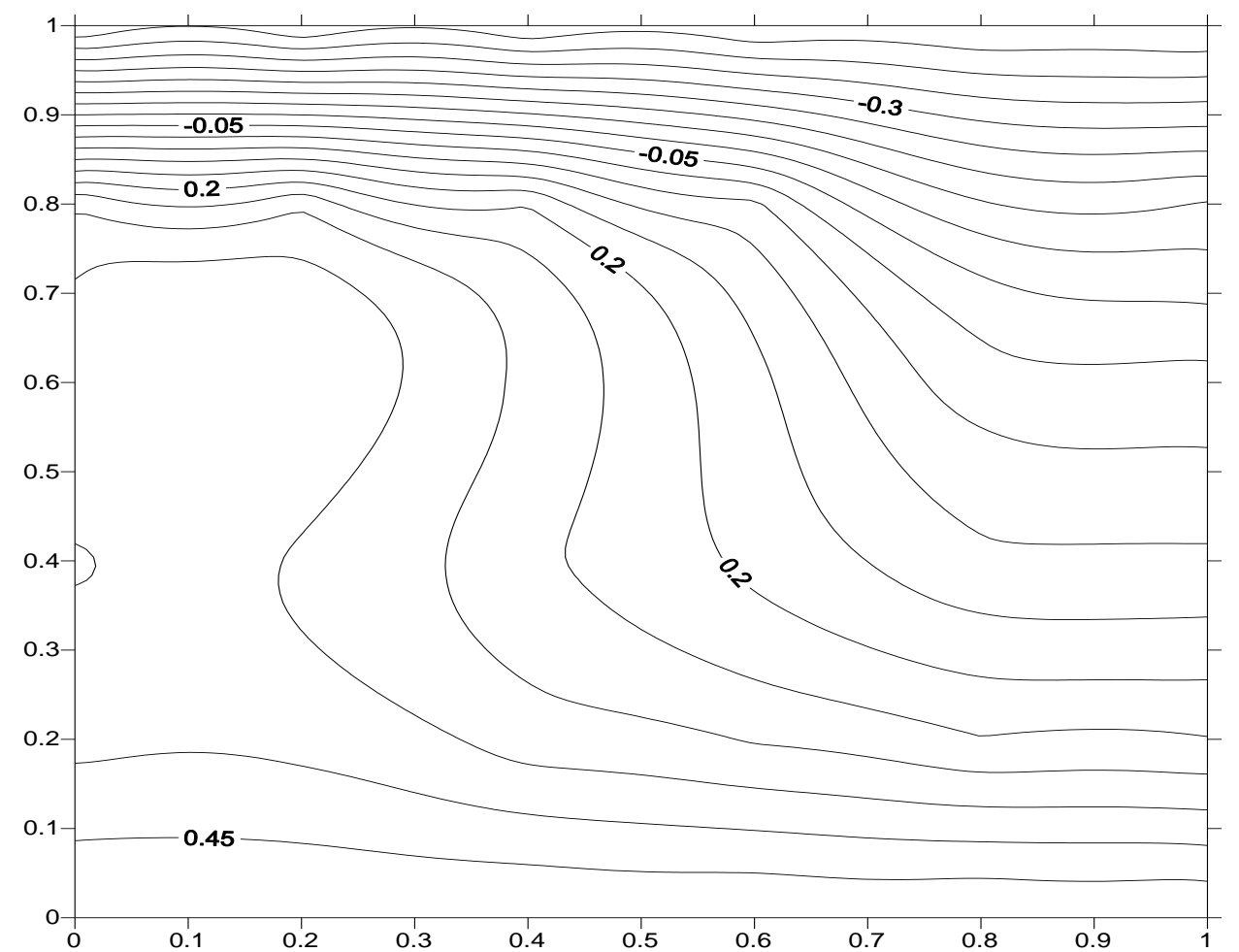

Figure 5d: : Iso-concentration profile for $\mathrm{Le}=1.0$

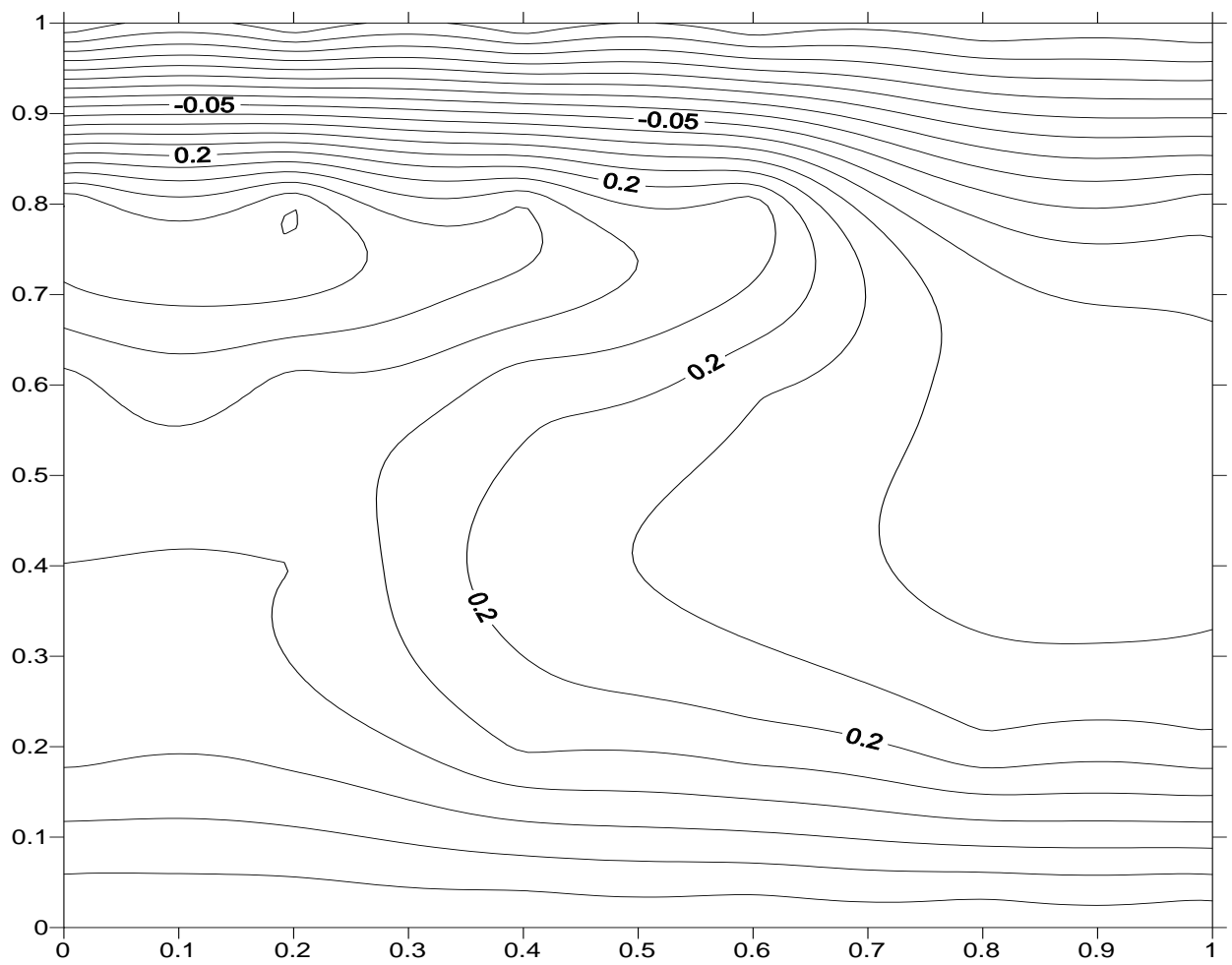

Figure 5d: : Iso-concentration profile for $L e=2.0$

Figures 5 a to $d$ : $R a=100, L e=1, B=1 ., \lambda=1 ., R=1 ., N=1$ 


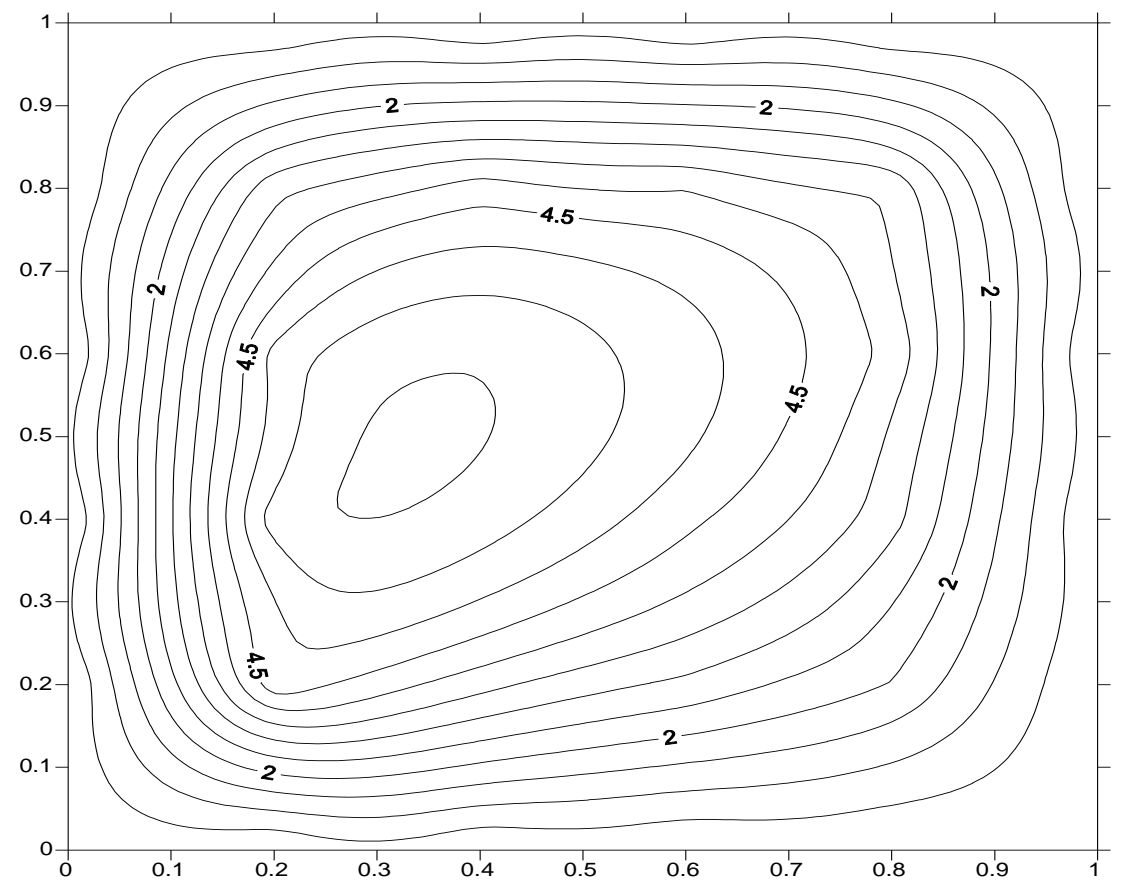

Figure 6a: Streamline profiles ( $R a=100, L e=1, B=1 ., \lambda=1 ., R=1 ., N=.01$ )

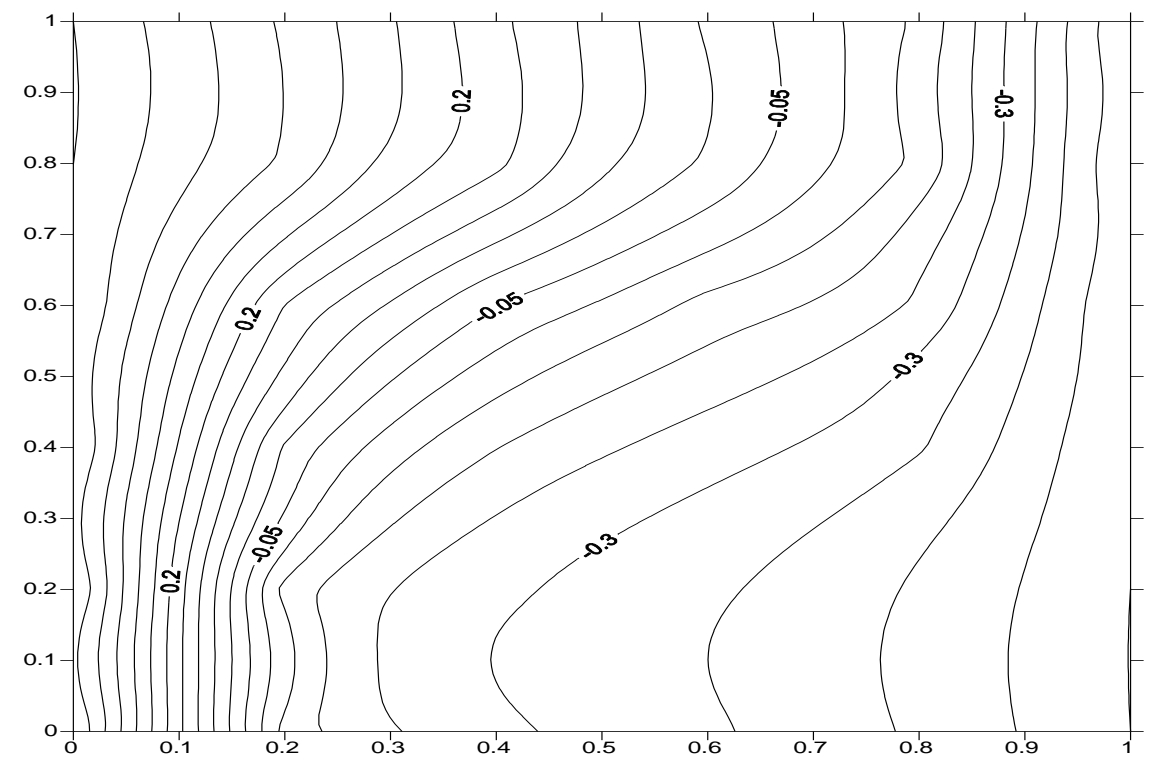

Figure 6b: Iso-temperature profiles ( $R a=100, L e=1, B=1 ., \lambda=1 ., R=1 ., N=.01$ ) 


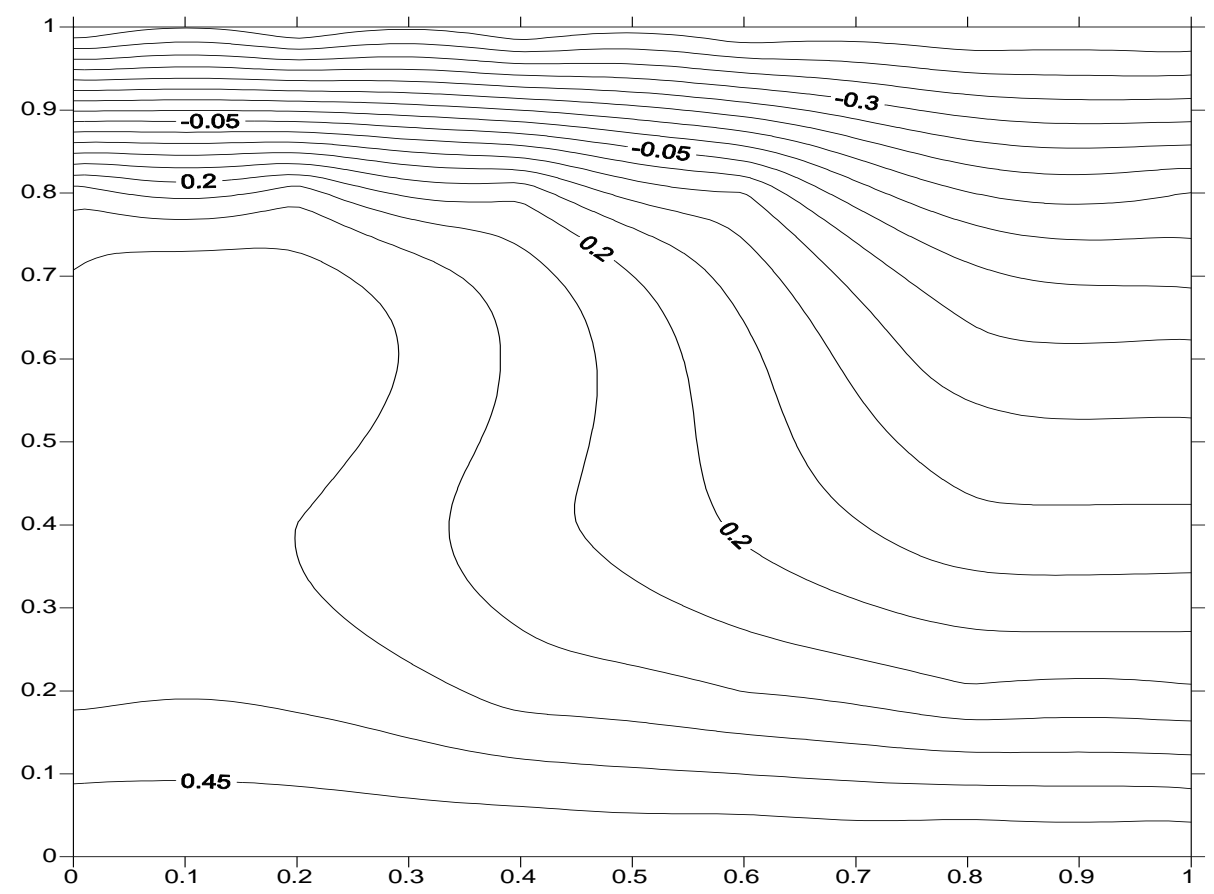

Figure 6c: Iso-concentration profiles $(R a=100, L e=1, B=1 ., \lambda=1 ., R=1 ., N=.01)$

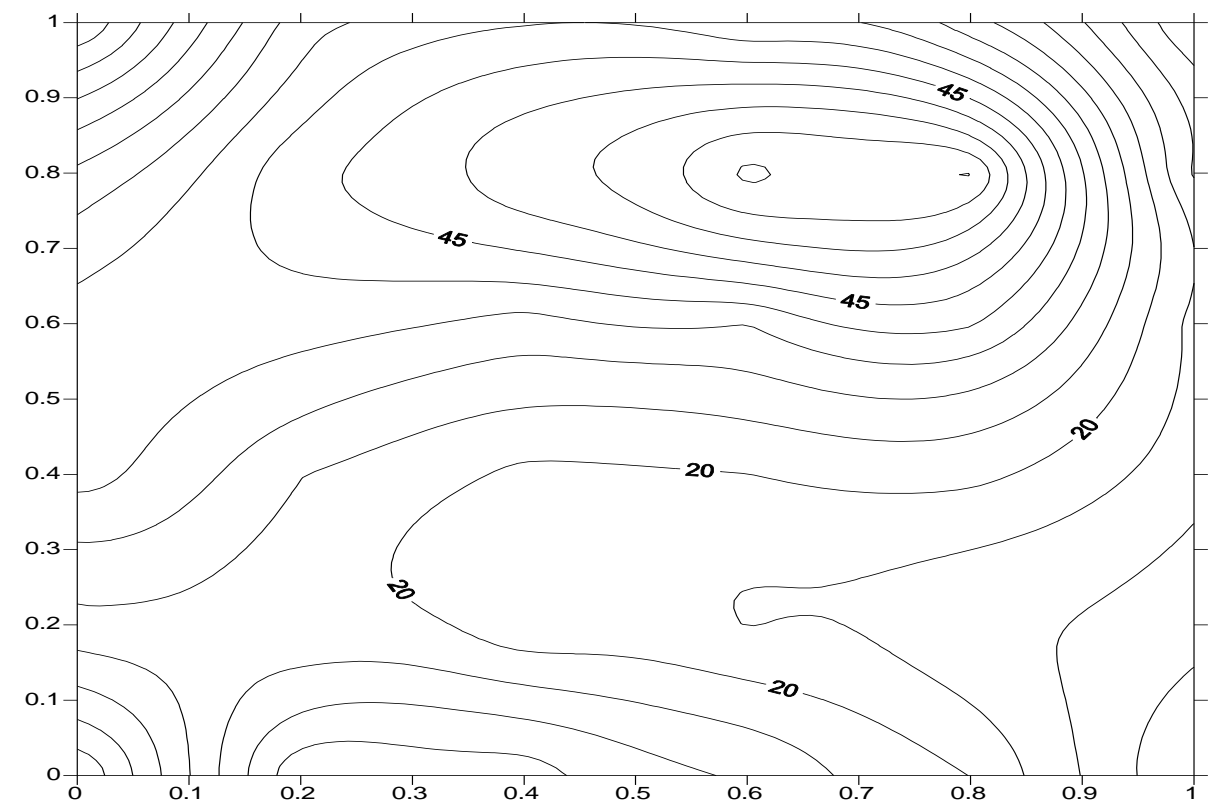

Figure 6 d: Iso-microrotation profiles $R a=100, L e=1, B=1 ., \lambda=1 ., R=1 ., N=.01$ ) 


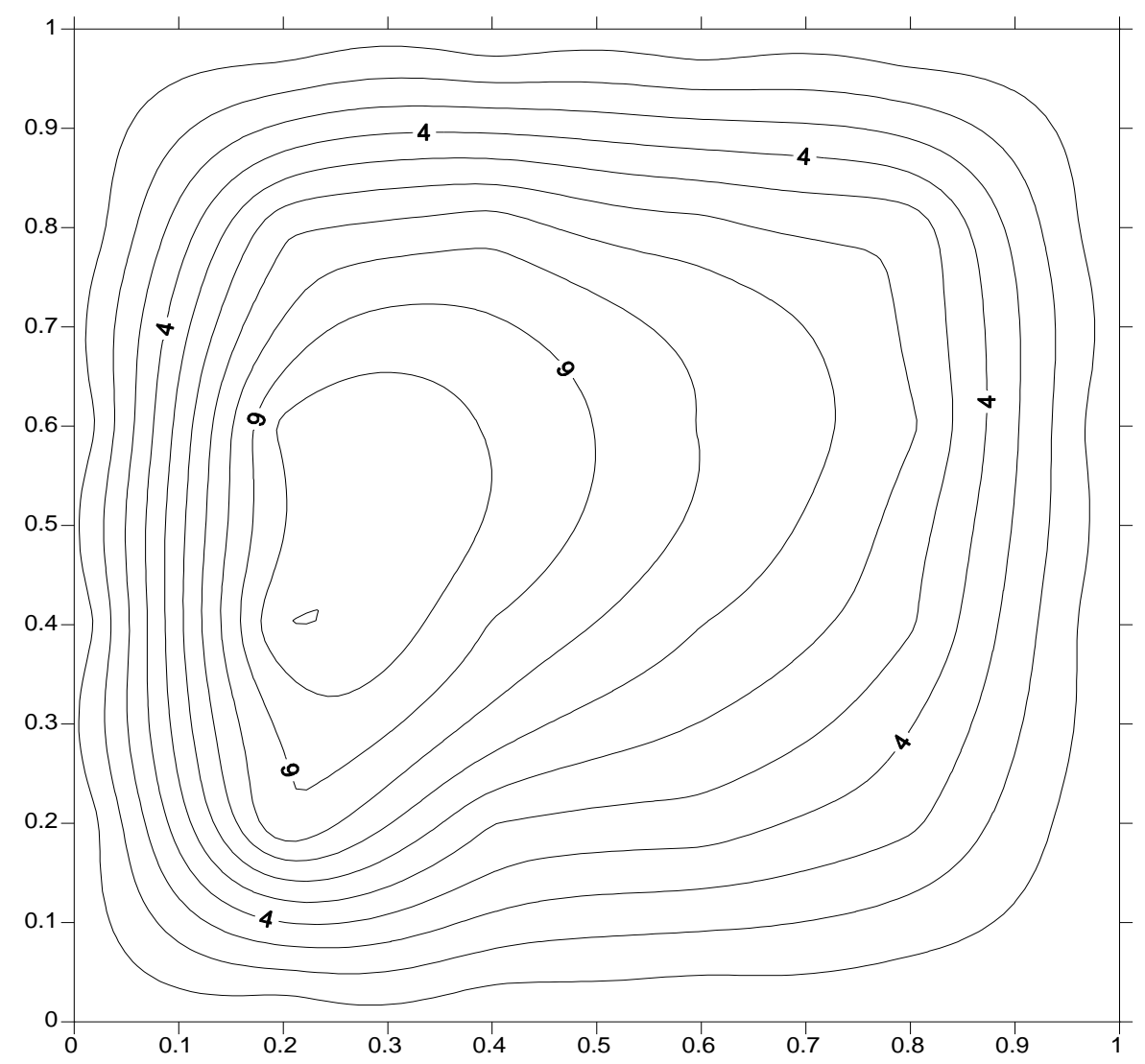

Figure 7a: Streamline Profiles ( $R a=200, L e=1, B=1 ., \lambda=1 ., R=1 ., N=.01)$

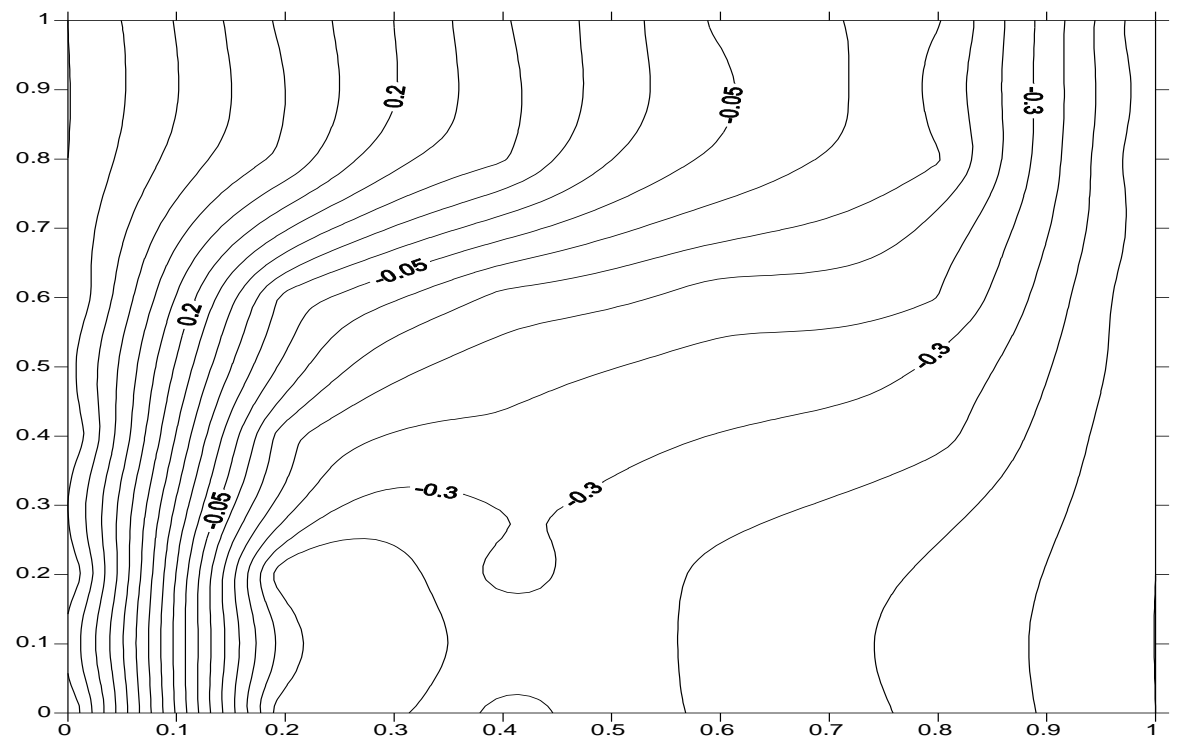

Figure 7b: Iso-temperature Profiles ( $R a=200, L e=1, B=1 ., \lambda=1 ., R=1 ., N=.01$ ) 


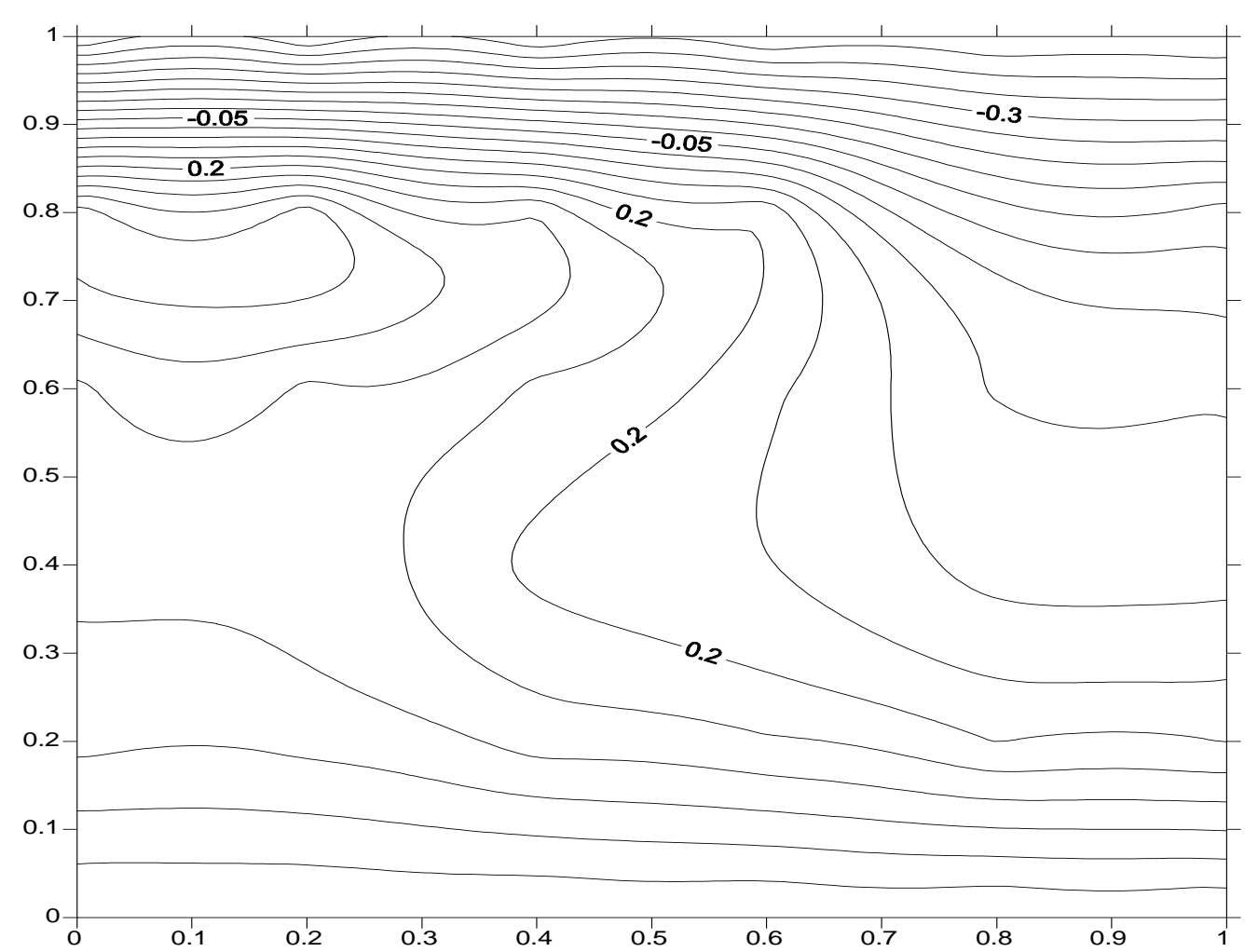

Figure 7c: Iso-concentration Profiles ( $R a=200, L e=1, B=1 ., \lambda=1 ., R=1 ., N=.01$ )

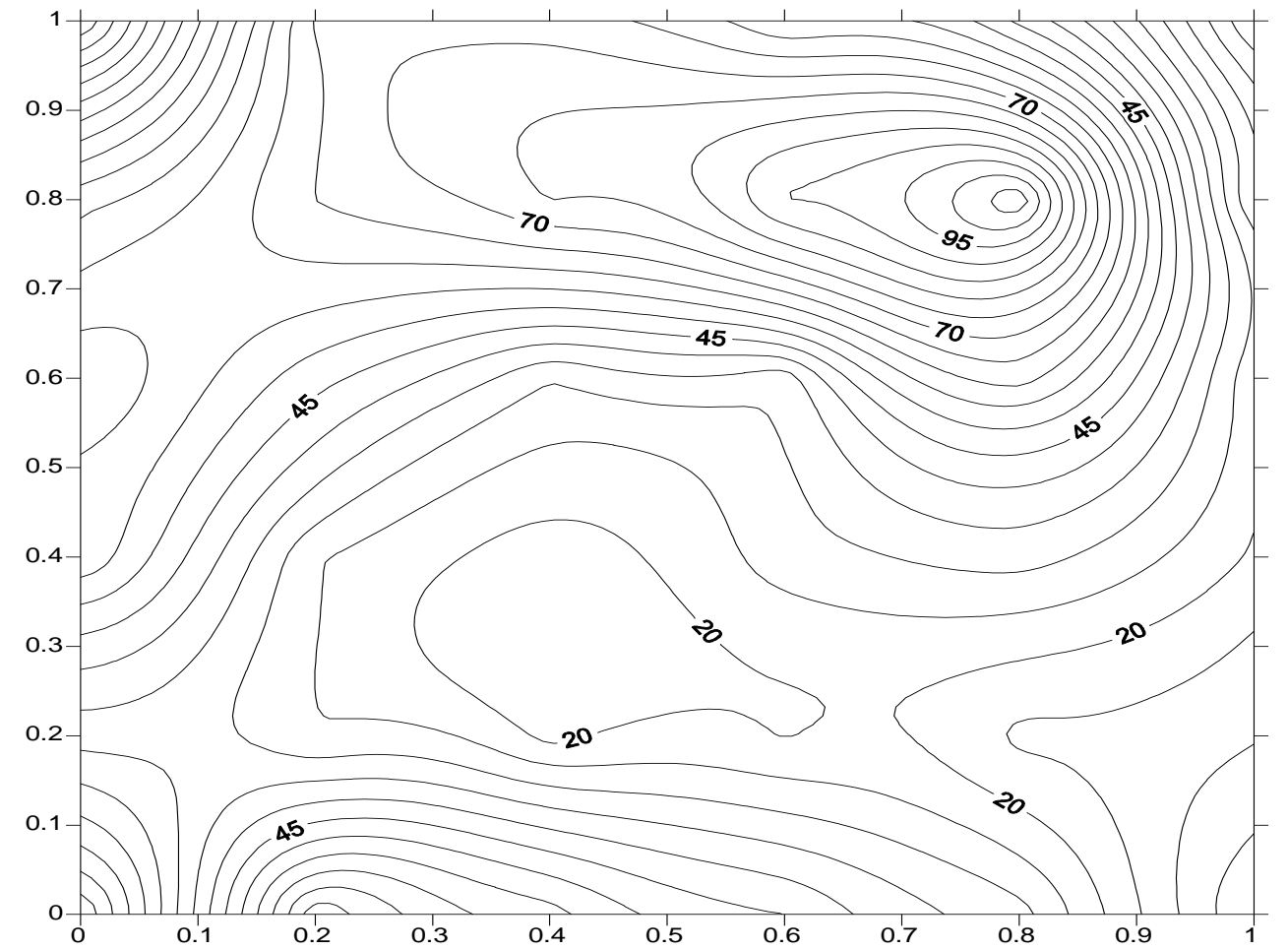

Figure 7d: Isomicrorotation Profiles ( $R a=200, L e=1, B=1 ., \lambda=1 ., R=1 ., N=.01$ ) 


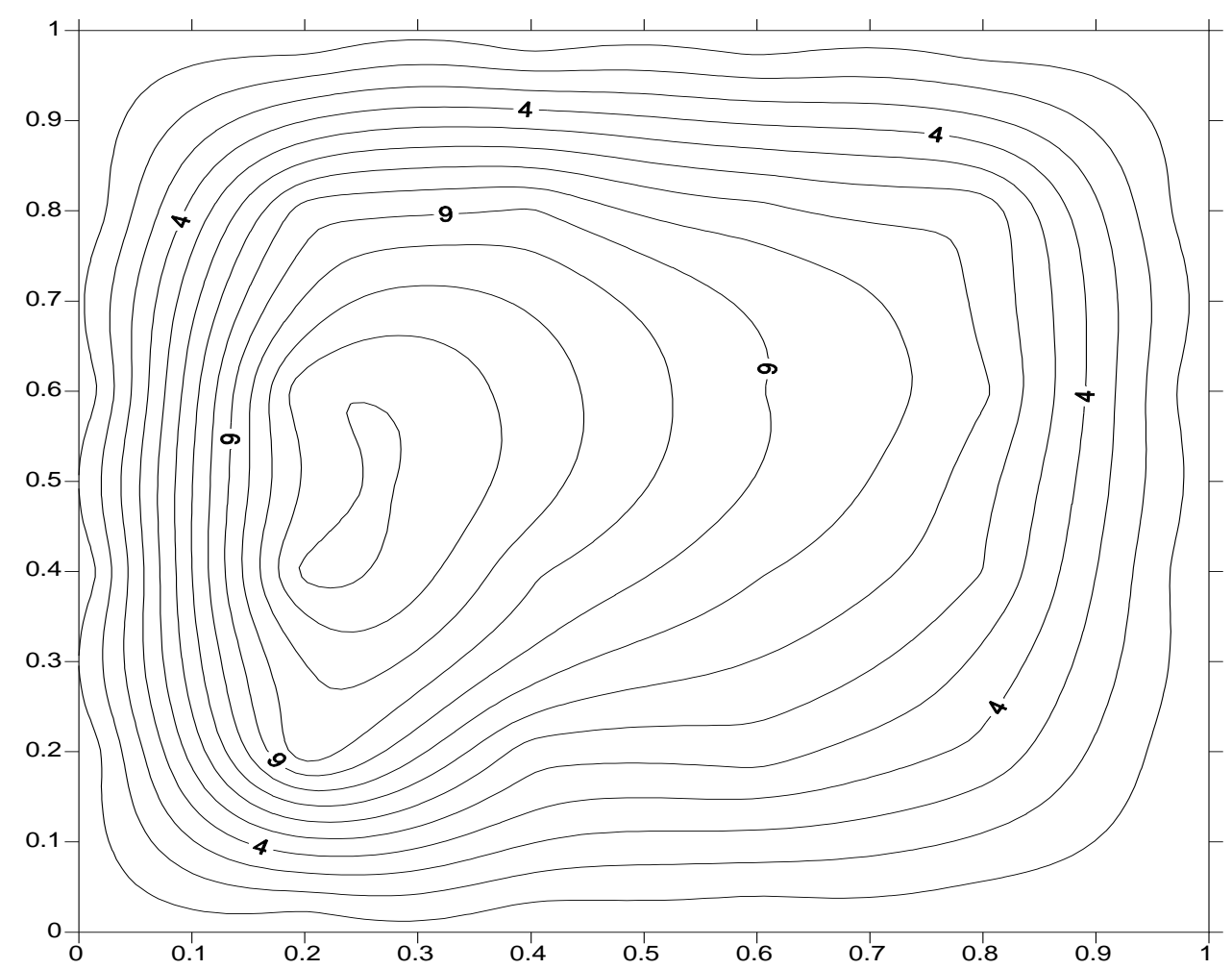

Figure 8a: Streamline profiles ( $R a=300, L e=1, B=1 ., \lambda=1 ., R=1 ., N=.01)$

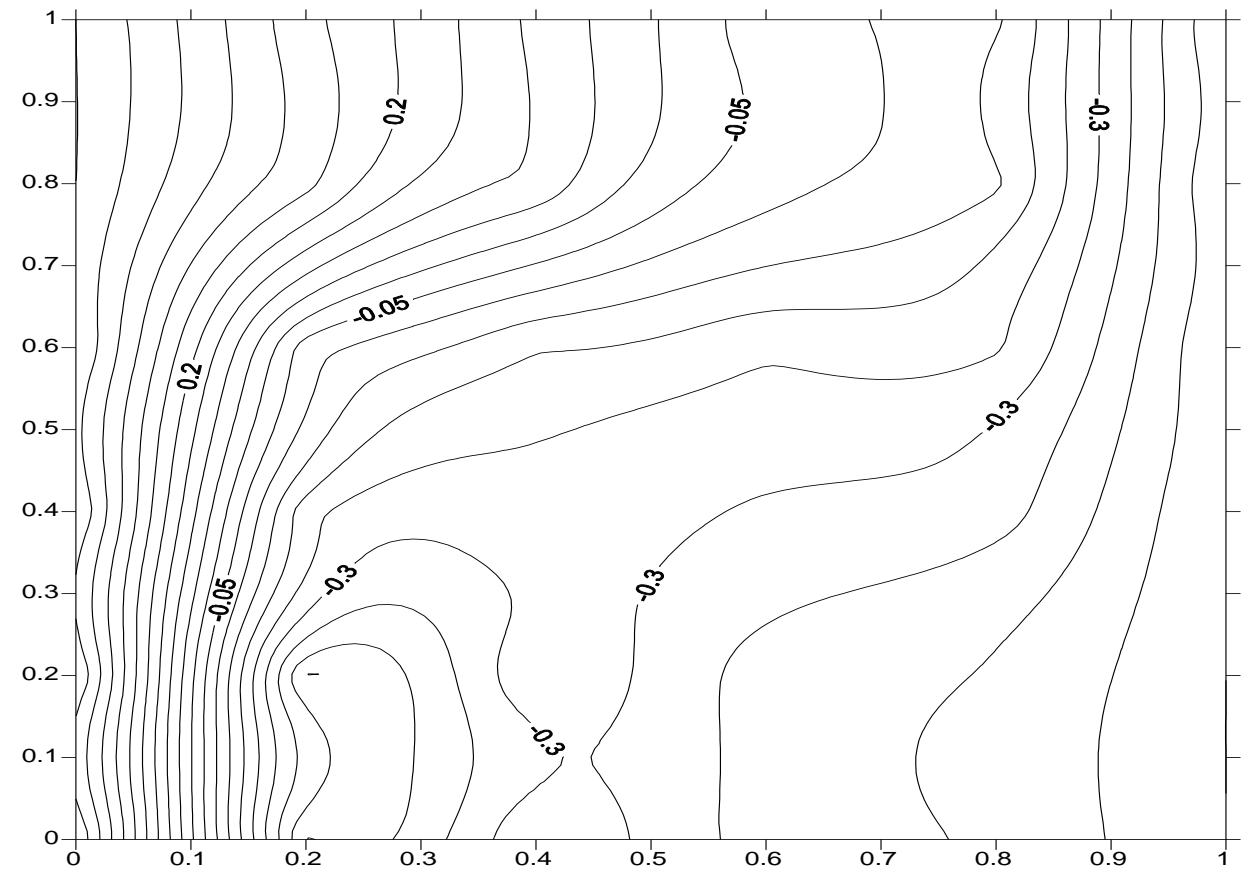

Figure 8b: Iso-temperature profiles ( $R a=300, L e=1, B=1 ., \lambda=1 ., R=1 ., N=.01$ ) 


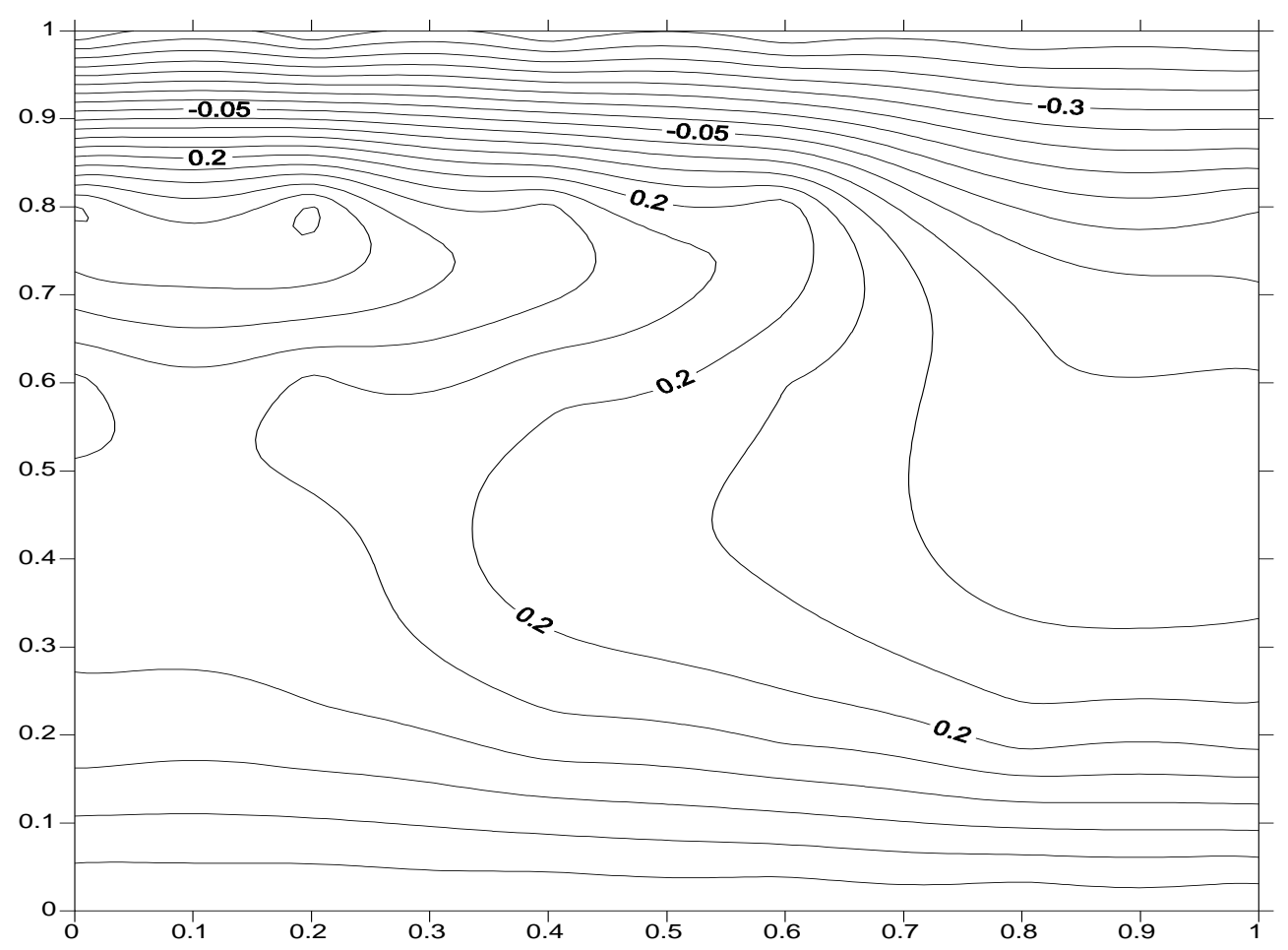

Figure 8c: Iso-concentration profiles ( $R a=300, L e=1, B=1 ., \lambda=1 ., R=1 ., N=.01)$

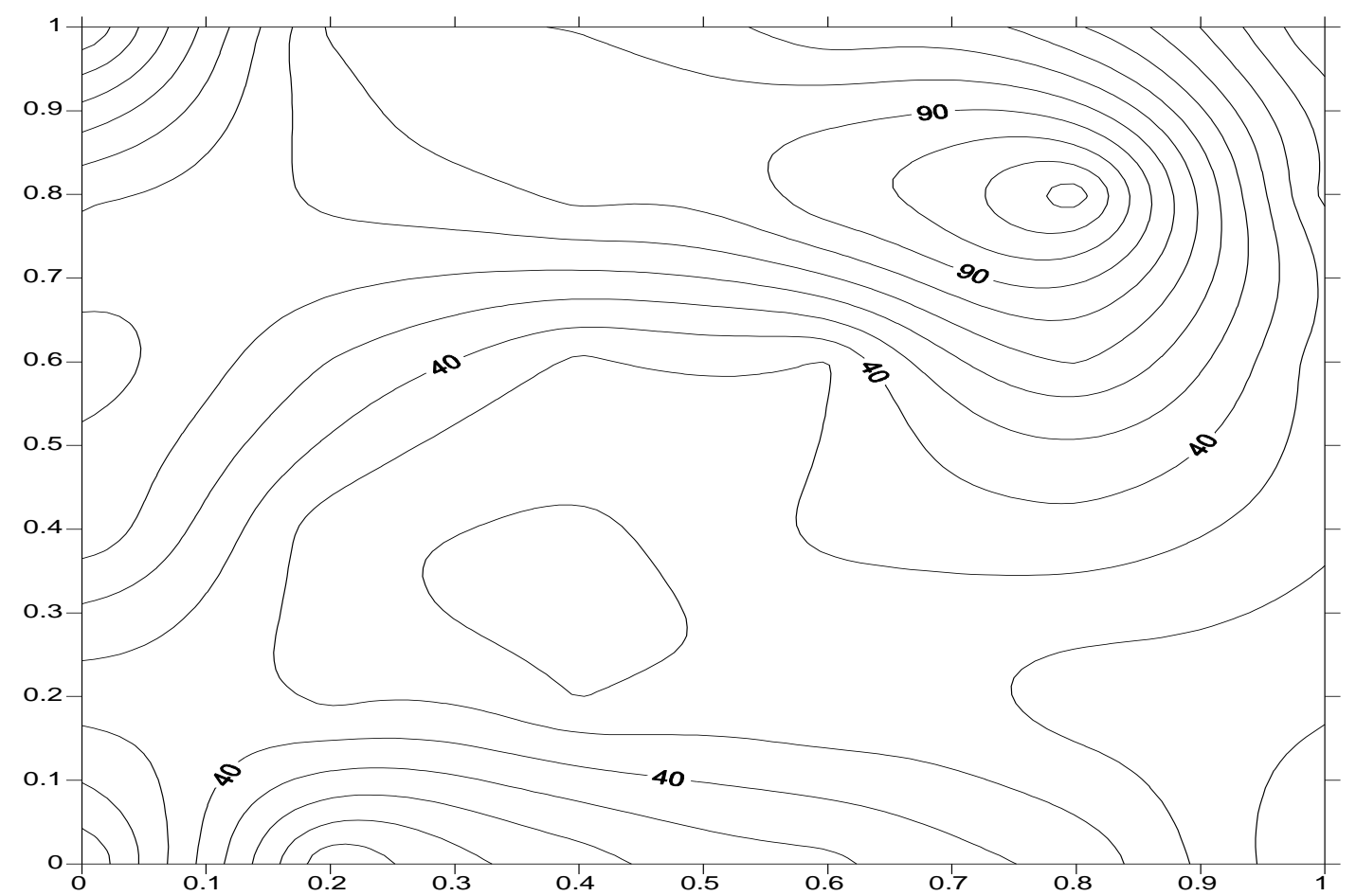

Figure 8d: Iso-micro-rotation profiles $(R a=300, L e=1, B=1 ., \lambda=1 ., R=1 ., N=.01)$ 


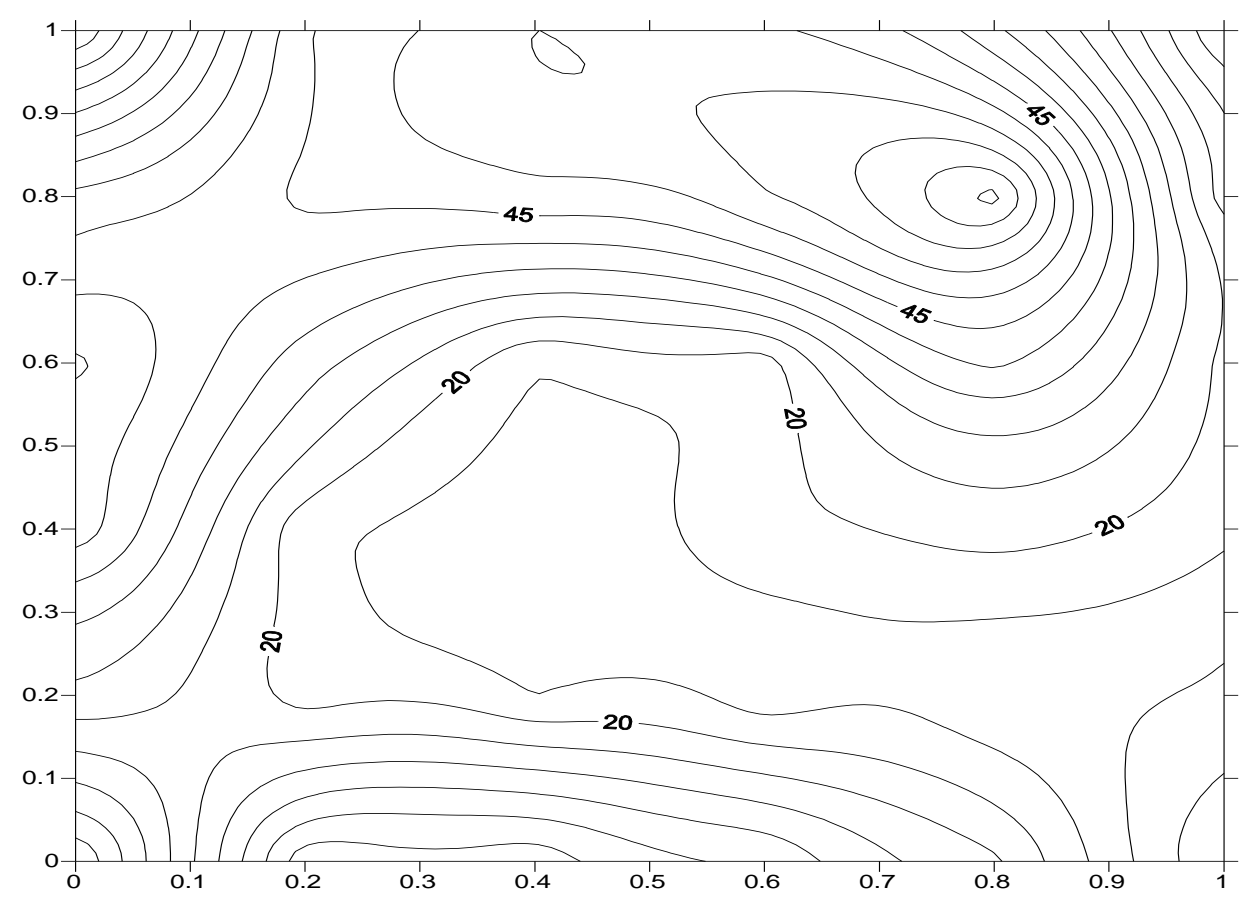

Figure 9a: Iso-microrotation for $\operatorname{Pr}=.733(R a=100, L e=1, B=1 ., \lambda=1 ., R=1 ., N=1)$

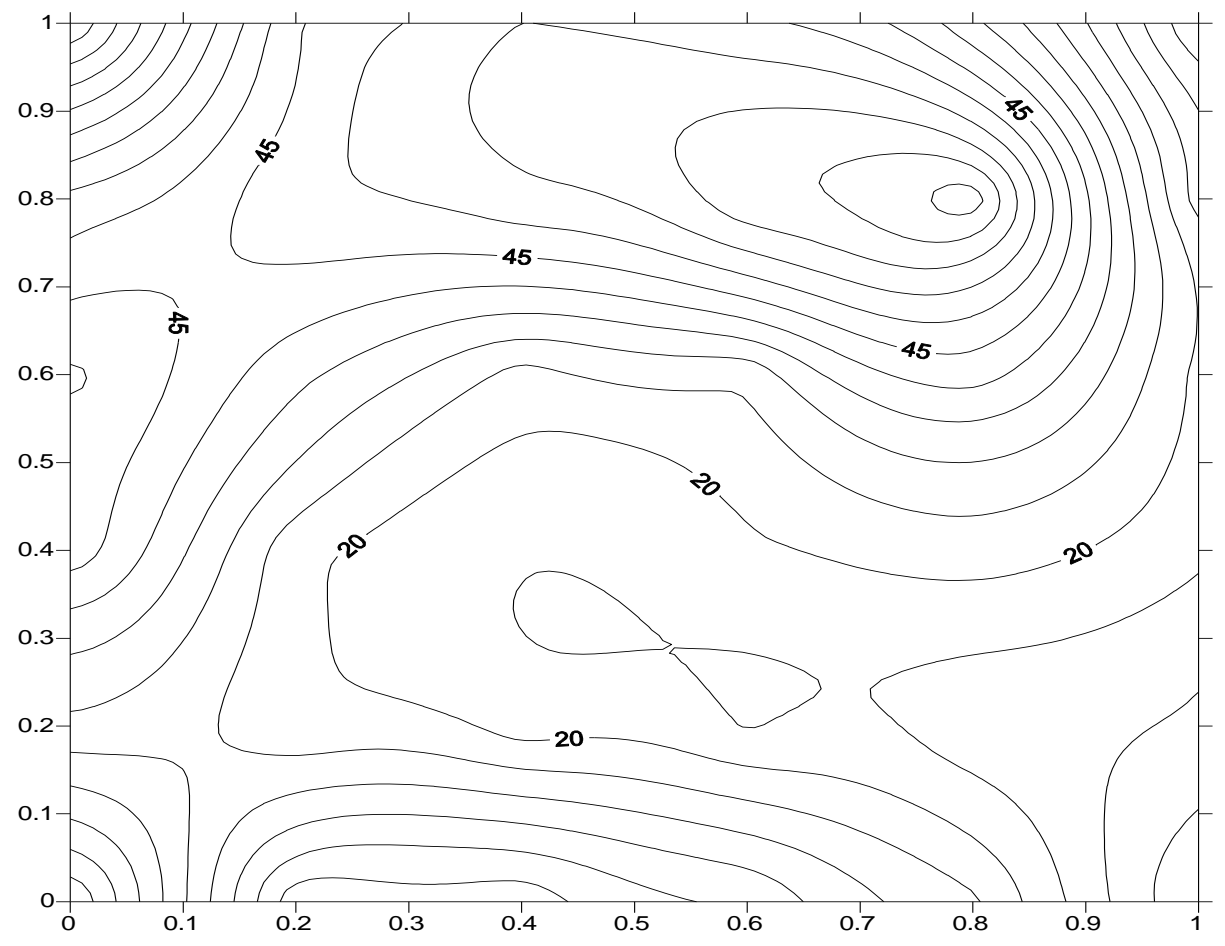

Figure 9b: Iso-microrotation for $\operatorname{Pr}=1.0(R a=100, L e=1, B=1, \lambda=1,, R=1 ., N=1)$ 


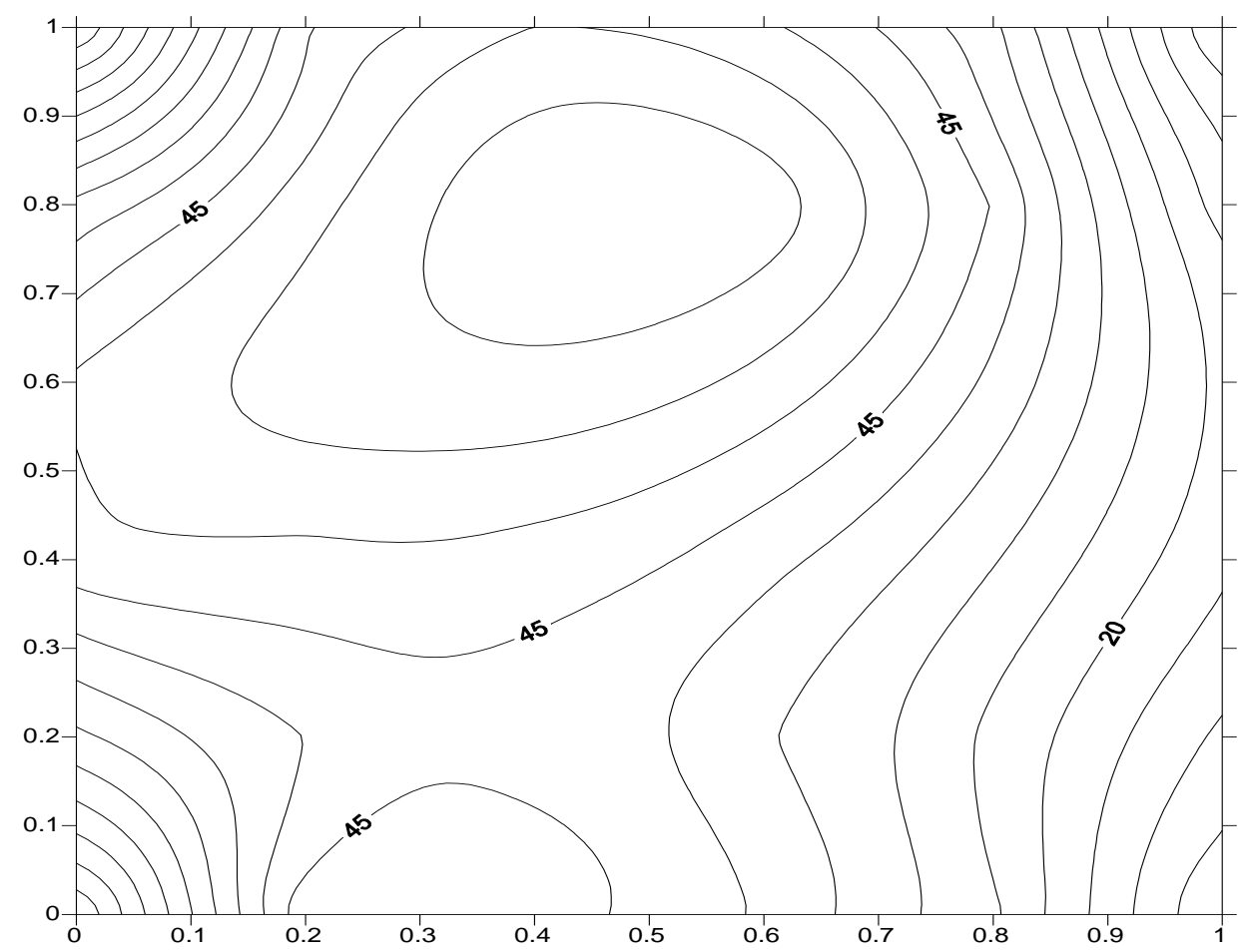

Figure 9c: Iso-microrotation for $\operatorname{Pr}=7(R a=100, L e=1, B=1 ., \lambda=1 ., R=1 ., N=1)$

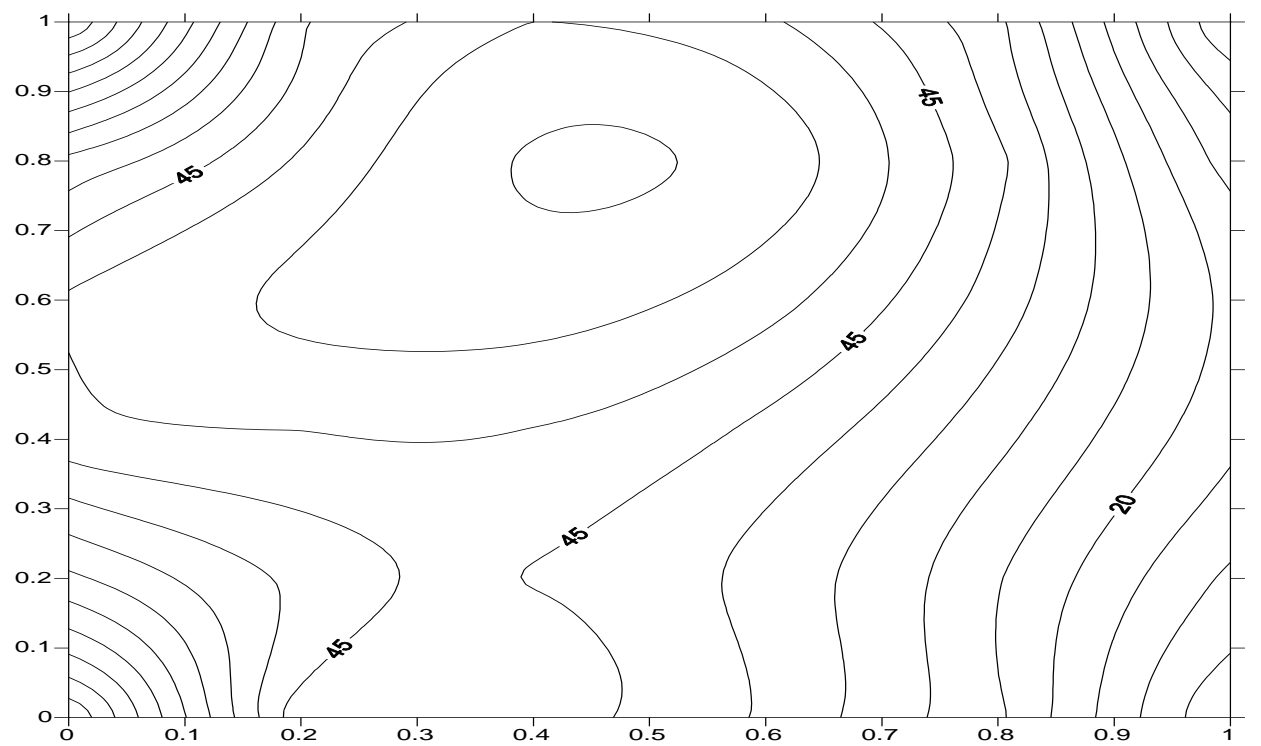

Figure 9d: Iso-microrotation for $\operatorname{Pr}=10(R a=100, L e=1, B=1 ., \lambda=1 ., R=1 ., N=1)$ 


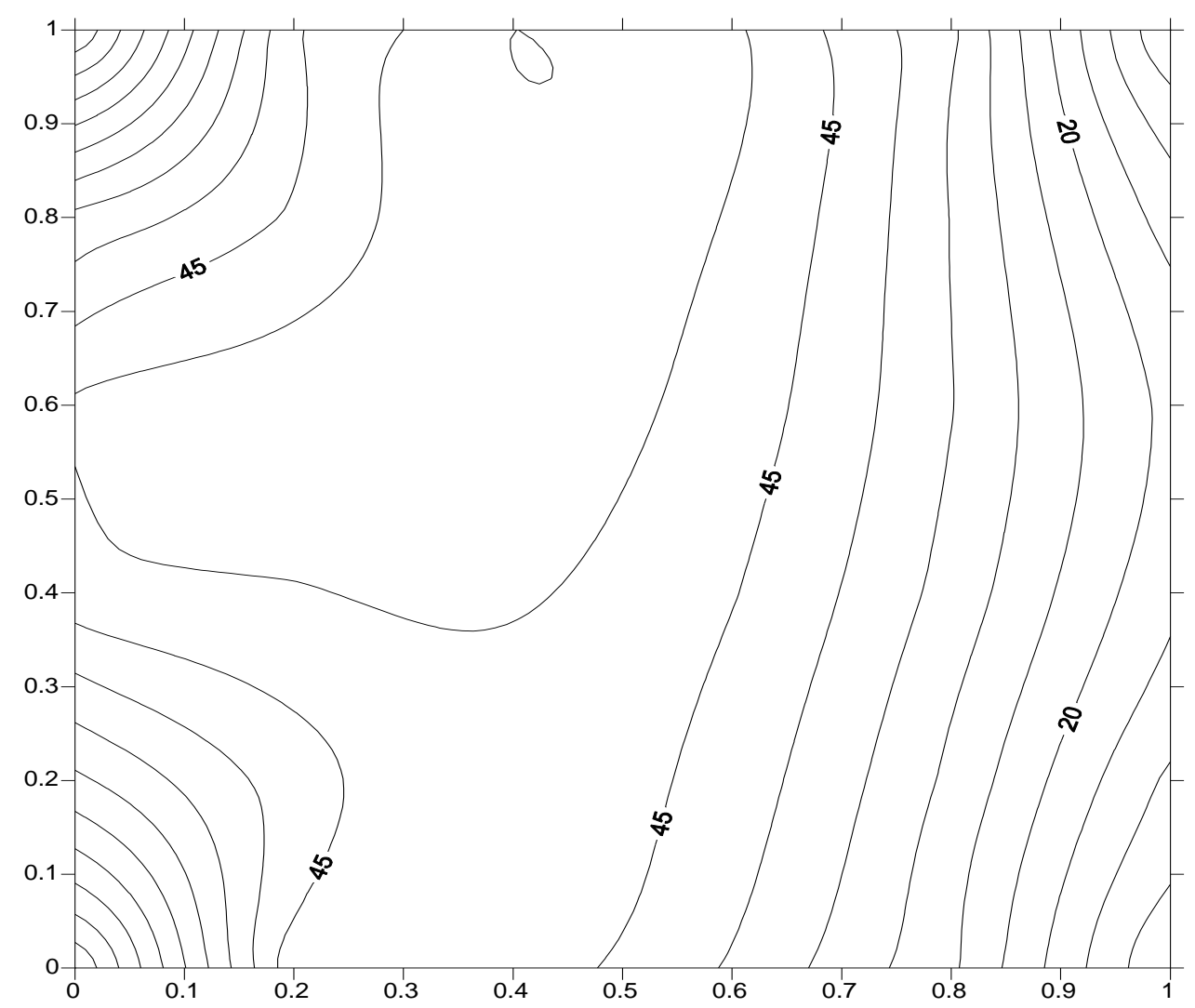

Figure 9e : Iso-microrotation for $\operatorname{Pr}=100(R a=100, L e=1, B=1 ., \lambda=1, R=1 ., N=1)$

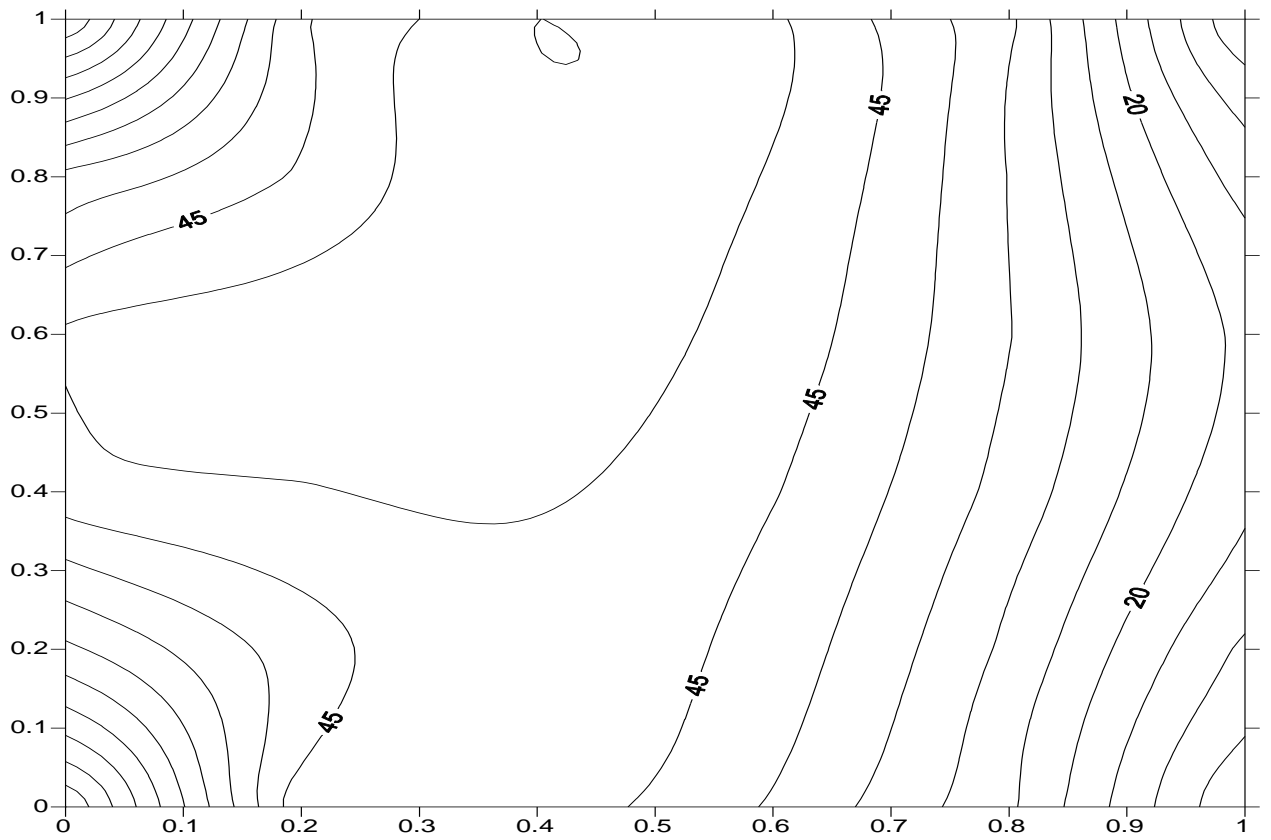

Figure 9f: : Iso-microrotation for $\operatorname{Pr}=1000(R a=100, L e=1, B=1 ., \lambda=1 ., R=1 ., N=1)$ 
The variation of the rate of heat transfer $\left(-\mathrm{T}^{\prime}(0)\right)$ and mass transfer $\left(-\mathrm{S}^{\prime}(0)\right)$ with respect to buoyancy parameter $(N)$ and Lewis number $(L e)$ are provided in table 1 . Results from both the finite element computations and the finite difference computations are shown to be in excellent agreement. It can be seen that the rate of heat transfer increases with increase in $N$, while rate of mass transfer first decreases for $N \leq 1$, but increases for $N>1$. The rate of heat transfer increases and mass transfer both decreases for $L e \leq 1$, while opposite effects are observed for $L e>1$. In all the data computed, $R a=100, \lambda=0.5, \operatorname{Pr}=1.0, R=1$ (micropolar vortex viscosity and Newtonian dynamic viscosity are equal) and $B=0.5$.

In the present study the flow in the enclosure comprises a single roll, the tendency of which is to rearrange the biofluid into a position of stable stratification, one in which the warm fluid that initially occupied the left half eventually moves to the upper half of the domain. Concentration gradient reversal is evident at the core of cavity due to strong flow recirculation, as shown in figure 1c. The isotherms reveal that the rate of heat transfer is high at the bottom of the hot wall due to flow impingement at this location, and rate of heat transfer decreases along the hot wall. Iso-concentration plots indicate a concentration bias towards the upper horizontal boundary while the micro-rotation profiles (figure 1d) show a clear vortex structure in the centre of the enclosure.

Figures 2a to $2 d$ illustrate the results obtained by increasing $N$ from zero to unity for which the solutal and thermal buoyancy are equal. Lewis number is unity and therefore the thermal and species diffusivities are also equal. For this value of $N$ there is a competition between thermal and solutal buoyancy forces. With the increase in buoyancy ratio the flow near the hot right wall is driven vertically upward, and whereas the low concentration at the upper wall causes the bioreactor fluid near it to sink. Clearly both the thermal and solutal buoyancy effects augment each other and thus they simultaneously accelerate the flow counterclockwise. As $N$ increases, the flow is driven solely by the solutal buoyancy forces. We note that for $\mathrm{N}=1$, the iso-temperature curves become more intensely packed towards the left wall of the enclosure (figure $2 \mathrm{~b}$ ) compared with the case for $N=0$ (figure $1 \mathrm{~b}$ ). The iso-concentration profiles are also substantially affected. They are intensified in the region of the upper horizontal wall and the vorticity near the central region of the enclosure is also increased (figure 2c) compared with the case for $\mathrm{N}=0$ (figure 1c). The micro-rotation profiles are also affected. Magnitudes in particular in the central zone of the enclosure are enhanced for $\mathrm{N}=1$ (figure 2d) compared with the case for $\mathrm{N}=0$ (figure 1d). 
The influence of yet larger values of buoyancy ratio $(\mathrm{N}=4)$ are depicted in figures 3a to 3d. It is clear from all the figures that for $\mathrm{N}=4$, the strength of the flow circulation is decreased (compared with lower $\mathrm{N}$ values in figures $1 \mathrm{a}$ to $1 \mathrm{~d}$ and $2 \mathrm{a}$ to $2 \mathrm{~d}$ ); conversely concentration and thermal reversal diminishes. Heat transfer takes place mainly by conduction as is evident from the isotherm distribution. Micro-rotation profiles are also seen to become more intensified with $\mathrm{N}=$ 4.

The typical feature of opposing double diffusion flow $(N<0)$ is shown in figures 4 a to 4d, for which the flow is driven mainly by destabilizing solutal buoyancy forces. In this sceanrio, the temperature and concentration are linearly stratified in the horizontal and vertical direction. The most striking features of the effect of negative $\mathrm{N}$ is the suppression of convection as a transport mechanism. The suppression is most dramatic in the vicinity of $N=-1$, i.e. in flows where the temperature and concentration buoyancy effects are of the same order of magnitude and in opposing direction. Indeed, the flow disappears altogether in the limiting case $L e=1$, $N=-1$. The circulation is reversed as $N$ because as low as $N=-4$ as shown in fig.4: here the flow is mainly counterclockwise, and the boundary layers start from the upper left hand and lower right hand corners. Micro-rotation is seen to weaken considerably in the central enclosure region (figure 4d).

Figures 5a to 5d illustrate the effect of Lewis number (Le) on the concentration field which is coupled to the heat transfer driven flow. Lewis number defines the ratio of thermal and mass diffusivities. It entails therefore the relative thickness of the thermal boundary layer thickness to the concentration boundary layer thickness. It also defines the ratio of Prandtl number to Schmidt number, where the latter is a relative measure of the effectiveness of momentum and mass transport by diffusion in the velocity and concentration boundary layers. At small Lewis numbers $(L e=0.1)$ the concentration boundary layers are no longer distinct and mass transfer through the porous layer is mainly by diffusion in the horizontal direction. The opposite effect is encountered at high Lewis numbers $(L e=5.0$ ), where the concentration boundary layers become sharper than the thermal boundary layers. In addition, at high Lewis numbers the mass diffusivity is low enough relative to the thermal diffusivity so that the horizontal intrusion layers lining the top and bottom walls are considerably sharper than their thermal counterparts. The net result is that the core of the concentration field at high Lewis numbers is in a state of almost uniform concentration.

The influence of Rayleigh number on the flow regime is illustrated in three sets of plots, figures $6 \mathrm{a}$ to $6 \mathrm{~d}(\mathrm{Ra}=100)$, figures $7 \mathrm{a}$ to $7 \mathrm{~d}(\mathrm{Ra}=200)$ and figures $8 \mathrm{a}$ to $8 \mathrm{~d}(\mathrm{Ra}=300)$. The other parameters in all three sets of distributions have values of $\mathrm{Le}=1, \mathrm{~B}=1, \lambda=1, \mathrm{R}=1$ and $\mathrm{N}=0.01$. The Rayleigh number signifies the relative importance of the buoyancy force to viscous force in 
mixed convection flows. As Ra increases from 100, to 200 and 300, streamline profiles (figures 6a, $7 \mathrm{a}$ and $8 \mathrm{a}$ ) become increasingly concentrated towards the left wall of the enclosure. Iso-temperature profiles are seen to intensify also in the vicinity of the left wall as Rayleigh number rises from 100, 200 to 300 as shown in figures $6 \mathrm{~b}, 7 \mathrm{~b}$ and $8 \mathrm{~b}$. Concentration profiles become increasingly intense towards the upper horizontal boundary as $R a$ increases (figures $6 \mathrm{c}, 7 \mathrm{c}$ and $8 \mathrm{c}$ ). Finally microrotation profiles are shown in figures $6 \mathrm{~d}, 7 \mathrm{~d}$ and $8 \mathrm{~d}$ as $R a$ increases from 100 to 200 and then 300 . A rise in $\mathrm{Ra}$ to 200 increases the concentration of micro-rotation in the top right zone of the enclosure while simultaneously intensifying the micro-rotation (angular velocities) in the central region. This trend however decreases as $R a$ is increased further to 200 indicating a critical zone between 200 and 300 where micro-rotation intensity is maximized.

The influence of Prandtl number on the micro-rotation profiles is illustrated in figures 9a to 9f. A rise in Pr from 0.733 (corresponding to air or water) through 1.0 (water), 7 (brine/water), 10 (weakly bio-rheological suspensions), 100 and 1000 (higher viscosity biofluids), corresponds to a decrease in thermal conductivity of the biofluid (i.e. an increase in Prandtl number for constant values of dynamic viscosity and specific heat capacity, $\operatorname{Pr}=\mathrm{c}_{\mathrm{p}} \mu / \mathrm{K}_{\mathrm{c}}$ ). Prandtl number quantifies the relative effectiveness of momentum and energy transport by diffusion in the velocity and thermal boundary layers. Prandtl therefore strongly influences the relative growth of the velocity and thermal boundary layers. Small values of the Prandtl number, $\operatorname{Pr}<<1$, means the thermal diffusivity dominates. Conversely with large values, $\operatorname{Pr}>>1$, the momentum diffusivity dominates the behavior. Prandtl number is inversely proportional to thermal conductivity of the biofluid. Therefore as Pr increases, thermal conductivity of the fluid must decrease and this reduces the contribution of thermal conduction heat transfer. Furthermore with increasing Prandtl number, the viscous boundary layer becomes increasingly larger than the thermal boundary layer. Prandtl number therefore is a significant parameter influencing thermofluid characteristics in the regime.

The other parameters in all six profiles have values of $R a=100, L e=1, B=1, \lambda=1, R=1$ and $N=1$. As $\operatorname{Pr}$ increases from 0.733 to 1.0 (figures $9 \mathrm{a}$ and $9 \mathrm{~b}$ ) we observe a decrease in concentration of is-micro-rotations in the upper right corner vicinity of the enclosure; there is nevertheless an increase in concentration of profiles in the central region. As Pr rises to 7 (figure 9c), profiles become less concentrated but then increase again for $\operatorname{Pr}$ of 10 (figure 9d). For very high values of $\mathrm{Pr}$, corresponding to lower thermal conductivities, profiles become progressively more sparse, even though magnitudes remain essentially the same. In the right half of the enclosure iso-micro-rotations are almost parallel with larger separations for the $\operatorname{Pr}=1000$ case. 


\section{CONCLUSIONS}

A two-technique numerical solution has been obtained for the micropolar double-diffusive heat and mass transfer in an enclosure, as a model of bioreactor systems. The results have indicated that:

1. For sufficiently small $N$, the flow is mainly governed by the thermal buoyancy, while for its large value, the flow characteristics are similar to those of pure solutal convection. The flow is solutally driven when $L e$ is small and thermally driven when $L e$ is sufficiently high. For intermediate value of $L e$, both solutions are possible.

2. The rate of heat transfer increases with increase in $N$, while mass transfer first decreases, then increases as $N$ increases.

3. The rates of heat and mass transfer are conversely affected with a rise in Lewis number.

4. Rising Prandtl number generally decreases micro-rotation concentrations in the enclosure.

5. An increase in Rayleigh number also increases streamlines circulation profiles towards the left wall of the enclosure, intensifies iso-temperature profiles in the vicinity of the left wall, enhances is-concentration (species) profiles towards the upper horizontal boundary and boosts concentration of iso-micro-rotations in the top right zone of the enclosure.

The present study has demonstrated the excellent versatility of finite element methods in simulating transport in bioreactor enclosures. Future studies will also incorporate chemical reaction effects. Reactive flows may be either homogenous or heterogenous, destructive or productive. They may also be first, second or higher order. These aspects may be of relevance to better understanding bioreactor physico-chemical micropolar flows since chemical reaction may significantly influence both heat and species diffusion rates. Indeed we have employed many of these approaches for simulating chemical reactions in recent years, and the approaches developed in [32]-[38] will be explored in enclosure micropolar convection flows in the near future. The formulations adopted will range from linear to quadratic and also exponential. They can be elegantly integrated into the transport equations.

\section{REFERENCES}

[1]K. Vant Riet and J. Tramper, Basic Bioreactor Design, Marcel Dekker, New York (1991). 
[2] A.I. Galaction, Cascaval, D., Oniscu, C. and Turnea, M. Prediction of oxygen mass transfer coefficients in stirred bioreactors for bacteria, yeasts and fungus broths. Biochemical Engineering Journal, 20, 85-94 (2004).

[3] D. A. Mitchell, O. F. von Meien, N. Krieger, Recent developments in modeling of solid-state fermentation: heat and mass transfer in bioreactors, Biochemical Engineering J., 13, 137-147 (2003).

[4] R. Van Kaam, D. Anne-Archard, M. Alliet Gaubert, C. Albasi, Rheological characterization of mixed liquor in a submerged membrane bioreactor: Interest for process management, J. Membrane Science, 317, 26-33 (2008).

[5] Ostrach, S., Natural convection with combined driving forces, Physico-Chemical Hydrodynamics J., 1, 233-247 (1980).

[6] Makham, B. I. and Rosenberger, F., Diffusive convection vapour transport across horizontal and inclined rectangular enclosures, J. Crystal Growth, 67, 241-254 (1984).

[7] Bergman, T.L., Incropera, F.P. and Viskanta, R., Correlation of mixed layer growth in a double-diffusive, salt-stratified system heated from below, ASME J. Heat Transfer, 108, 206-211 (1986).

[8] Raganathan, P. and Viskanta, R., Natural convection in a square cavity due to combined driving forces, Numerical Heat Transfer, 14, 35-59 (1988).

[9] Nishimura, T., Imoto, T. and Miyashita, H., Occurrence and development of double-diffusive convection during solidification of a binary system, Int. J. Heat and Mass Transfer, 37, 14551464 (1994).

[10] Bejan, A. and Trevisan O.V., Mass and heat transfer by high Rayleigh number convection in a porous medium heated from below, Int. J. Heat Mass Transfer, 30, 2341-2356 (1987).

[11] Lin K.W., Unsteady natural convection heat and mass transfer in a saturated porous enclosure, Heat and Mass Transfer J., 28, 49-56,(1993).

[12] Mohamad, A.A. and Bennacer R., Double-diffusion natural convection in an enclosure filled with saturated porous medium subjected to cross gradients, Int. J. Heat Mass Transfer, 45, 37253740 (2002).

[13].Mohamad, A.A. and Bennacer R., Natural convection in a confined saturated porous medium with horizontal temperature and vertical solutal gradients, Int. J. Thermal Sci., 40, 82-93 (2001). 
[14] Khanafer, K. and Chamkha, A. J., Mixed convection flow in a lid-driven enclosure filled with a fluid-saturated porous medium, Int. J. Heat Mass Transfer, 42, 2465-2481 (1999).

[15] Khanafer, K. and Vafai, K., Double-diffusive mixed convection flow in a lid-driven enclosure filled with a fluid-saturated porous medium, Numerical Heat Transfer, 42, 465-486 (2002).

[16] Eringen A.C., Theory of micropolar fluids, J. Math. Mech., 16, 1-18 (1966).

[17] Jena S.K., Bhattacharyya S.P., The effect of microstructure on the thermal convection in a rectangular box of fluid heated from below, Int. J. Engineering Science, 24, 69-76 (1986).

[18] Chen C.K., Hsu T.H., Natural convection of micropolar fluids in an enclosure, ASME/JSME Thermal Engineering Conference Proc., Tokyo, Japan, 1, 199-205 (1991).

[19] Hsu,T. H. and Chen, C.K., Natural convection of micropolar fluids in a rectangular enclosure, Int. J. Engineering Science, 34, 4, 407-415 (1996).

[20] Chiu,C.P., Shich,J.Y. and Chen,W.R., Transient natural convection of micropolar fluids in concentric spherical annuli, Acta Mechanica, 132, 75-92 (1999).

[21] J. Srinivas and J. V. Ramana Murthy, Second law analysis of the flow of two immiscible micropolar fluids between two porous beds, J. Eng. Thermophy., 25(1), 126--142 (2016).

[22] J. V. Ramana Murthy, J. Srinivas and K. S. Sai, Flow of immiscible micropolar fluids between two porous beds, J. Porous Media, 17 (4), 287-300 (2014).

[23] Eringen A.C, Micro-Continuum Field Theories: Volume II- Fluid Media, Springer, New York (2001).

[24] Reddy J. N., An Introduction to the Finite Element Method, McGraw-Hill Book Co., New York (1985).

[25] O. Anwar Bég, Tasveer A. Bég, R. Bhargava, S. Rawat and D. Tripathi, Finite element study of pulsatile magneto-hemodynamic non-Newtonian flow and drug diffusion in a porous medium channel, J. Mechanics in Medicine and Biology, 12 (4) 1250081.1 - 1250081.26 (2012).

[26] P. Rana, R. Bhargava and O. Anwar Bég, Finite element modeling of conjugate mixed convection flow of $\mathrm{Al}_{2} \mathrm{O}_{3}$-water nanofluid from an inclined slender hollow cylinder, Physica Scripta, 88 (2013) 15pp

[27] V. Rajesh, O. Anwar Bég, MHD transient nanofluid flow and heat transfer from a moving vertical cylinder with temperature oscillation, Computational Thermal Sciences, 6, 439-450 (2014). 
[28] R. Bhargava, S. Sharma, O. Anwar Bég and Zueco, J, Finite element study of nonlinear twodimensional deoxygenated biomagnetic micropolar flow, Communications in Nonlinear Science and Numerical Simulation, 15, 1210-1233 (2010).

[29] Andersen, D. A. et al, Computational Fluid Mechanics and Heat Transfer, Hemisphere, Washington, USA (1984).

[30] Sohail, A., H. A. Wajid and M. M. Rashidi, Numerical modeling of capillary-gravity waves using the phase field method, Surface Review and Letters, 21.03, 1450036 (2014).

[31] Sohail, A., M. J. Uddin, and M. M. Rashidi, Numerical study of free convective flow of a nanofluid over a chemically reactive porous flat vertical plate with a second-order slip model, ASCE J. Aerospace Engineering (2015): 04015047.

[32] O. Anwar Bég, R. Bhargava, S. Rawat, H. S. Takhar and T. A. Bég, A study of buoyancydriven dissipative micropolar free convection heat and mass transfer in a Darcian porous medium with chemical reaction, Nonlinear Analysis: Modeling and Control J., 12, 2, 157-180 (2007).

[33] M.M. Rashidi, M. Ferdows, Md. Jashim Uddin, O. Anwar Bég and N. Rahimzadeh, Group theory and differential transform analysis of mixed convective heat and mass transfer from a horizontal surface with chemical reaction effects, Chemical Engineering Communications, 199:8, 1012-1043 (2012).

[34] O. Anwar Bég, M.M. Rashidi, M. Keimanesh and Tasveer A. Bég, Semi-numerical modelling of "chemically-frozen" combusting buoyancy-driven boundary layer flow along an inclined surface, Int. J. Applied Math. Mech., 9(1): 1 - 16 (2013).

[35] O. Anwar Bég, S.S. Motsa, M.N. Islam and M. Lockwood, Pseudo-spectral and variational iteration simulation of exothermically-reacting Rivlin-Ericksen viscoelastic flow and heat transfer in a rocket propulsion duct, Computational Thermal Sciences, 6, 1, 1-12 (12 pages) (2014).

[36] V. Rajesh, O. Anwar Bég, Ch. Sridevi and K. Jonah Philliph, Finite element analysis of unsteady MHD free convective laminar boundary-layer accelerated dissipative flow with uniform suction and chemical reaction, Int. J. Energy \& Technology, 6, 1-10 (2014).

[37] M. J. Uddin, O. Anwar Bég, A. Aziz and A. I. M. Ismail, Group analysis of free convection flow of a magnetic nanofluid with chemical reaction, Math. Prob. Engineering 2015, Article ID 621503, 11 pp (2015). doi:10.1155/2015/621503.

[38] G. Ravi Kiran, G. Radhakrishnamacharya and O. Anwar Bég, Peristaltic flow and hydrodynamic dispersion of a reactive micropolar fluid: simulation of chemical effects in the digestive process, J. Mechanics in Medicine and Biology (2016). DOI: 10.1142/S0219519417500130

\section{NOMENCLATURE}

\section{Roman}

A enclosure aspect ratio

B micro-inertia density parameter

D species (molecular) diffusivity 
g gravitational acceleration

$G$ ' dimensional micro-rotation component (angular velocity)

$G \quad$ non-dimensional micro-rotation component (angular velocity)

he $\quad x$-direction step-length in finite difference algorithm

$H^{\prime} \quad$ height of enclosure

$K_{v} \quad$ Eringen micropolar parameter

$\left[K^{m n}\right]$ stiffness matrix in finite element domain

ke $\quad y$-direction step-length in finite difference algorithm

Le Lewis number

$L^{\prime} \quad$ width of enclosure

$M \quad$ angular velocity (microrotation) vector

$N \quad$ buoyancy ratio

$\mathrm{Nu} \quad$ Nusselt number

$P \quad$ hydrodynamic pressure

Pr Prandtl number

$R \quad$ Eringen non-dimensional micropolar vortex viscosity parameter

Ra Rayleigh number

Sh Sherwood number

$\Delta S^{\prime} \quad$ concentration difference

$S_{h}{ }^{\prime} \quad$ concentration at lower boundary of enclosure

$S_{1}{ }^{\prime} \quad$ concentration at upper boundary of enclosure

$S_{0}{ }^{\prime} \quad$ reference concentration at $x^{\prime}=0, y^{\prime}=0$.

$S$ ' dimensional concentration

$S$ non-dimensional concentration

$T_{h}{ }^{\prime} \quad$ temperature at vertical left wall of enclosure

$T c^{\prime} \quad$ temperature at vertical right wall of enclosure

$T_{0}{ }^{\prime} \quad$ reference temperature at $x^{\prime}=0, y^{\prime}=0$.

$\Delta T, \quad$ temperature difference

$T$, dimensional temperature

$T \quad$ non-dimensional temperature

$u$ ' dimensional $x$ '-direction velocity

$u \quad$ non-dimensional $x$ '-direction velocity

$v^{\prime} \quad$ dimensional $y^{\prime}$-direction velocity

$v \quad$ non-dimensional y'-direction velocity

$V \quad$ translational velocity vector

$w_{i} \quad$ arbitrary test function in finite element model

$x^{\prime} \quad$ coordinate parallel to base of enclosure (horizontal)

$y^{\prime} \quad$ coordinate transverse to base of enclosure (vertical)

\section{Greek}

$\alpha^{*} \quad$ viscosity coefficient of micropolar fluid

$\alpha \quad$ thermal diffusivity of micropolar fluid

$\beta \quad$ viscosity coefficient of micropolar fluid

$\gamma \quad$ viscosity coefficient of micropolar fluid 
$\lambda \quad$ viscosity coefficient of micropolar fluid (micropolar material parameter)

$\mu \quad$ viscosity coefficient of micropolar fluid (dynamic viscosity)

$\chi \quad$ viscosity coefficient of micropolar fluid

$\rho \quad$ mass density of micropolar fluid

$\beta_{S} \quad$ coefficient of species expansion

$\beta_{T} \quad$ coefficient of thermal expansion

$\psi_{i} \quad$ linear interpolation function in finite element model

$\Omega^{e} \quad$ rectangular element in finite element discretized domain

$v \quad$ kinematic viscosity

$\varphi^{\prime} \quad$ dimensional stream function

$\varphi \quad$ non-dimensional stream function 